\title{
NUEVAS EXPRESIONES DE LAS NTCM (2017) PARA EL CÁLCULO DE LA RESISTENCIA DE MUROS DIAFRAGMA DE MAMPOSTERÍA
}

\author{
J. Martin Leal Graciano ${ }^{(1)}$, J. J. Pérez-Gavilán E. ${ }^{(2)}$, J. Humberto Castorena González ${ }^{(3)}$, Alfredo Reyes \\ Salazar $^{(1)}$ y Manuel A. Barraza Guerrero ${ }^{(4)}$
}

\begin{abstract}
RESUMEN
Se presentan las nuevas expresiones para calcular la resistencia a corte de muros diafragma de mampostería incluida en el Capítulo 4 de las Normas Técnicas Complementarias para el Diseño y Construcción de Estructuras de Mampostería 2017 del Gobierno de la Ciudad de México. Se consideran tres modos de falla: por aplastamiento, por deslizamiento y por tensión diagonal. Las expresiones se adaptaron de las especificadas en el código canadiense, utilizando los parámetros de resistencia usados en la norma mexicana, y se refiere a una resistencia a corte en los tres modos de falla. Se verificó que los resultados de resistencia calculados con estas expresiones dan resultados satisfactorios, al compararlos con resultados de una campaña experimental que también se presentan aquí.

La campaña experimental incluyó seis especímenes, escala 1:2, en los que las variables de estudio fueron la rigidez relativa muro/marco, el uso de elementos de confinamiento y el refuerzo horizontal. Los resultados indican que la rigidez relativa muro/marco tiene un efecto relevante en la resistencia al agrietamiento del muro y la resistencia máxima del sistema. Adicionalmente, la contribución del refuerzo horizontal a la resistencia lateral depende de la rigidez relativa muro/marco, un fenómeno que las expresiones de diseño no toman en cuenta. Los elementos de confinamiento no incrementan la resistencia lateral ni la capacidad de desplazamiento del sistema; sin embargo, las dalas y castillos mejoran la estabilidad fuera del plano del muro y el contacto entre el muro y el marco.
\end{abstract}

Palabras Clave: mampostería; muros diafragma; confinamiento; refuerzo; resistencia

\footnotetext{
Artículo recibido el 5 de octubre de 2018 y aprobado para su publicación el 2 de julio de 2019. Se aceptarán comentarios y/o discusiones hasta cinco meses después de su publicación.

(1) Facultad de Ingeniería Culiacán, Universidad Autónoma de Sinaloa, Calzada Las Américas y Universitarios, Ciudad Universitaria s/n, 80040, Culiacán de Rosales, Sinaloa, México; jesusleal@uas.edu.mx; reyes@uas.edu.mx;

(2) Instituto de Ingeniería, Universidad Nacional Autónoma de México, Ciudad Universitaria, 04510, Ciudad de México, México, iipge@pumas.iingen.unam.mx

(3) Facultad de Ingeniería Mochis, Universidad Autónoma de Sinaloa, Fuente de Poseidón, s/n, 81210, Los Mochis, Sinaloa, México; humbertocastorena@yahoo.com.mx

(4) Facultad de Ingeniería, Arquitectura y Diseño, Universidad Autónoma de Baja California, Carretera Transpeninsular Ensenada - Tijuana 3917, Zona Playitas, 22860 Ensenada, Baja California, México; barraza.manuel@uabc.edu.mx
} 


\title{
NEW EXPRESSIONS ADOPTED BY MEXICAN CODE (NTCM, 2017) TO CALCULATE THE SHEAR STRENGTH OF INFILL MASONRY WALLS
}

\begin{abstract}
The new shear strength design expressions for infill-walls included in the 2017 release of the Mexican code are presented. Three failure modes are considered, crushing, sliding and diagonal tension. The expressions were adapted from those specified in the Canadian masonry code, to include the Mexican code strength parameters and to make the expressions refer, for the three failure modes, to a shear resistance. It was verified that the shear strength given by the new expressions is satisfactory after comparing their prediction to the results of an experimental campaign that is also presented herein.

The experimental investigation included six specimens, scaled $1: 2$, in which the study variables were the wall to frame stiffness ratio, the use of confining elements and horizontal reinforcement. The results indicate that the wall to frame stiffness ratio has an important effect on the cracking strength of the walls and the maximum shear strength of the system. Moreover, the contribution of horizontal reinforcement to the lateral strength depends on the wall to frame stiffness ratio, a phenomenon that the design expressions do not take into consideration. The confining elements surrounding the infill walls do not increase the lateral strength nor the displacement capacity of the system; however, they enhance the out of plane stability of the wall and the contact conditions between the wall and frame.
\end{abstract}

Keywords: masonry; infill wall; confinement; reinforcement; strength

\section{INTRODUCCIÓN}

Un muro diafragma es aquel muro que se encuentra rodeado por vigas y columnas de un marco estructural al que proporciona rigidez y resistencia ante cargas laterales (NTCM, 2017). A diferencia de los muros confinados, los muros diafragma no soportan cargas verticales debido a que las columnas son axialmente más rígidas que los muros. Otra diferencia con respecto a los muros confinados es que los muros diafragma se construyen después que los elementos del marco, generándose una junta fría entre ellos. Debido a que las características de deformación del muro y el marco son diferentes, estos elementos se separan, ante la acción de fuerzas laterales, en dos esquinas del muro diagonalmente opuestas, mientras que en las otras dos esquinas interactúan a lo largo de una cierta longitud de contacto (Figura 1). Cuando la carga lateral cambia de sentido, el mecanismo de arriostramiento del marco por el muro se desarrolla por compresión a largo de la otra diagonal, al igual que los efectos que se producen (Crisafulli, 1997).

Este comportamiento sugirió a los investigadores que el efecto del muro puede ser representado por un elemento diagonal en compresión axial que restringe la deformación lateral del marco. La idea fue primeramente propuesta por Polyakov en 1956 (Madia \& Parsekian, 2011). Después, Holmes (1961) propuso un modelo en el que el muro se remplaza por un elemento diagonal articulado en los extremos con las mismas propiedades mecánicas y espesor que el muro, y el ancho de la diagonal es igual a un tercio de la longitud diagonal del muro.

Posteriormente, algunos investigadores propusieron modelos más refinados. Stafford-Smith et al. (Stafford-Smith, 1962, 1966, 1967; Stafford-Smith y Carter, 1969) relacionaron el ancho de la diagonal equivalente con la longitud de contacto muro-marco, que a su vez depende de la rigidez relativa muro/marco dada por la ecuación 1 , 


$$
\lambda h=h\left(\frac{E_{m} t \sin 2 \theta_{d}}{4 E_{f} I_{c} H}\right)^{\frac{1}{4}}
$$

donde $h$ es la altura del marco medida al eje de la viga, $\theta_{d}$ es el ángulo de inclinación de la diagonal del muro con respecto a la horizontal, $E_{f}$ es el módulo de elasticidad del material usado en el marco, $I_{c}$ es el momento de inercia de la sección transversal de las columnas respecto al eje centroidal perpendicular al plano del marco, $E_{m}$ es el módulo de elasticidad de la mampostería y $t$ y $H$ son el espesor y la altura del muro (Figura 1). En la ecuación 1 se puede notar que valores crecientes de $\lambda h$ indican que el muro incrementa su rigidez respecto a la del marco. El modelo de Stafford-Smith (1962) es ampliamente reconocido. El código canadiense (CSA S304-14, 2014) propone utilizar el método de diagonal equivalente, basándose en dicho modelo para estimar las fuerzas en los muros diafragma.

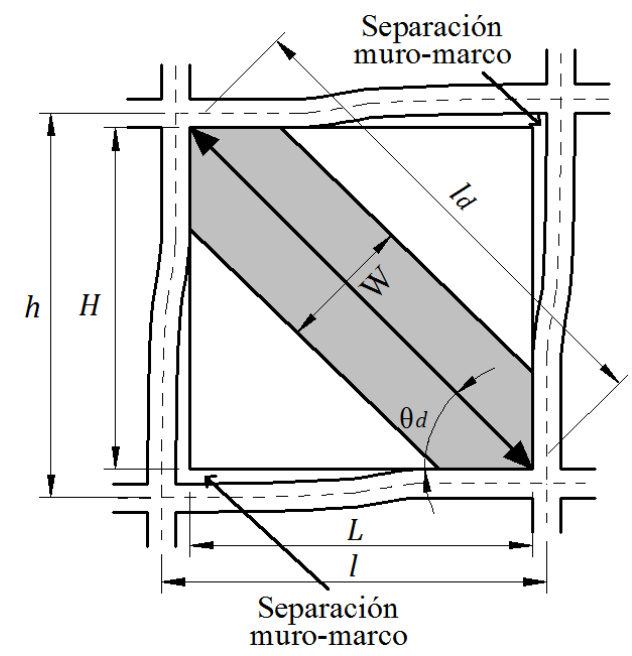

Figura 1. Configuración deformada de un marco relleno con un muro diafragma ante carga lateral y nomenclatura

Basado en resultados experimentales y analíticos, Mainstone (1974) propuso la ecuación 2 para el cálculo del ancho de la diagonal equivalente $(W)$, la cual está en función del parámetro de rigidez relativa de Stafford-Smith (1962) expresado en la ecuación 1. Esta ecuación se incluyó en FEMA-274 (FEMA, 1997) y FEMA-306 (FEMA, 1998) para el análisis y rehabilitación de construcciones (como lo reporta Asteris et al. 2011).

$$
W=\left[0.175(\lambda h)^{-0.4}\right] \cdot l_{d}
$$

En México, Bazán (1980) estableció la ecuación 3 para calcular el ancho de la diagonal, producto de correlacionar los resultados numéricos de un estudio paramétrico con elementos finitos de marcos rellenos de un nivel y una crujía.

$$
\mathrm{W}=(0.35+0.022 \cdot \beta) \cdot H
$$

En la ecuación 3, $\beta$ es un parámetro adimensional que expresa la rigidez relativa entre muro y marco definido en la ecuación 4.

$$
\beta=\frac{E_{f} \cdot A_{c}}{G_{m} \cdot A_{m}}
$$


donde $A_{c}$ es el área transversal bruta de las columnas, $G_{m}$ es el módulo de rigidez de la mampostería y $A_{m}$ es el área transversal del muro. Se observa que valores crecientes de $\beta$ indican que el marco incrementa su rigidez respecto a la del muro, contrariamente a lo establecido con el parámetro $\lambda h$.

En las normas norteamericanas (MSJC, 2010) también se establece un modelo basado en el concepto de la diagonal equivalente, en el cual el ancho de la diagonal se calcula mediante la ecuación 5. Esta ecuación es una interpretación de los análisis de Flanagan (2001), los cuales se realizaron a partir de datos experimentales.

$$
W=\frac{0.3}{\lambda h \cos \theta_{d}}
$$

En la ecuación $5, \lambda h$ y $\theta_{d}$ son las mismos parámetros expresados en la ecuación 1 . El código de Nueva Zelanda (NZS 4230, 2004) establece que el ancho de la diagonal equivalente se debe calcular igual a una cuarta parte de su longitud (ecuación 6)

$$
W=\frac{\sqrt{l^{2}+h^{2}}}{4}
$$

donde $l$ y $h$ son la longitud y altura del marco, como se define en la Figura 1.

Debido a la acción de cargas laterales, los marcos rellenos con muros diafragma puede desarrollar diferentes modos de falla, los cuales se asocian a un patrón de daño. Producto de investigaciones experimentales y analíticas (por ejemplo, Mehrabi y Shing, 1996; El-Dakhakhni et al. 2003; Asteris et al. 2011) realizadas en los últimos 60 años, se han identificado cinco distintos modos de falla de marcos rellenos con muros diafragma:

1) Falla por deslizamiento (Figura 2a). La falla se genera a lo largo de un plano horizontal en las juntas de mortero, ocasionando la separación del muro en dos o más partes. Se produce en muros largos con relación de aspecto $H / L<1$, que da lugar a esfuerzos bajos en el sentido perpendicular a las juntas. La separación del muro provoca la libre deformación de las columnas, lo cual a su vez ocasiona que se formen articulaciones plásticas en ellas. Si el plano de deslizamiento se forma cerca de la altura media de las columnas, puede provocar el efecto de columna corta en el marco que, eventualmente, puede conducir al colapso debido a una falla por cortante (Figura 2a).

2) Aplastamiento de las esquinas cargadas del muro (Figura 2b). Este modo de falla se produce debido a los elevados esfuerzos de compresión biaxial en esas zonas. Este tipo de falla ocurre con mayor frecuencia cuando los muros se construyen con mampostería de baja resistencia a compresión y el marco es flexible ya que la longitud de contacto en las esquinas es menor que cuando el marco es robusto. Adicionalmente, este tipo de falla es más frecuente en muros esbeltos, esto es, con $H / L>1$. Esto se debe a que en ese caso la componente vertical de la fuerza en el muro es considerable, lo que produce fuerzas de fricción mayores evitando la falla por deslizamiento.

3) Falla por compresión de la diagonal (Figura 2b). Consiste en el aplastamiento de la mampostería en la región central del muro. Este modo de falla se asocia a muros largos (diagonales esbeltas), los cuales se pandean fuera del plano. 
4) Falla por tensión diagonal. Este modo de falla se caracteriza por la aparición de grietas a lo largo de la diagonal en compresión y comúnmente ocurre junto a una falla por deslizamiento, como se observa en la Figura 2a.

5) Falla en el marco (Figura 2a). Consiste en la formación de articulaciones plásticas en las columnas del marco o en la conexión viga-columna. Este modo de falla se asocia a marcos débiles.

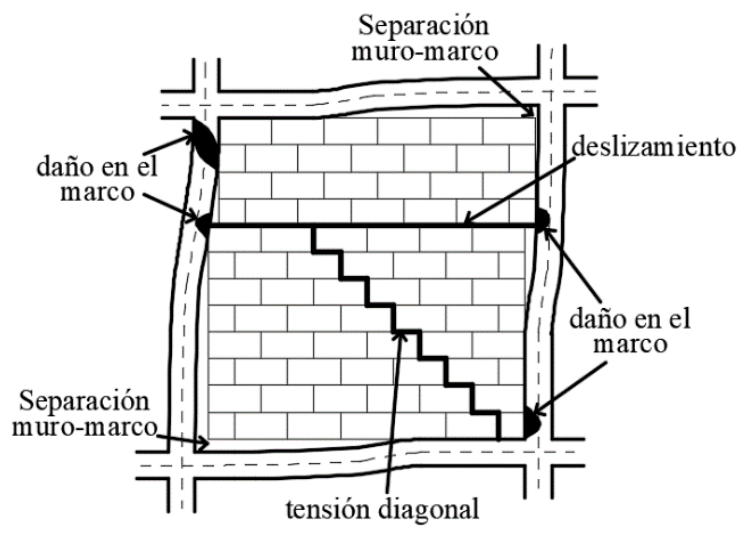

a)

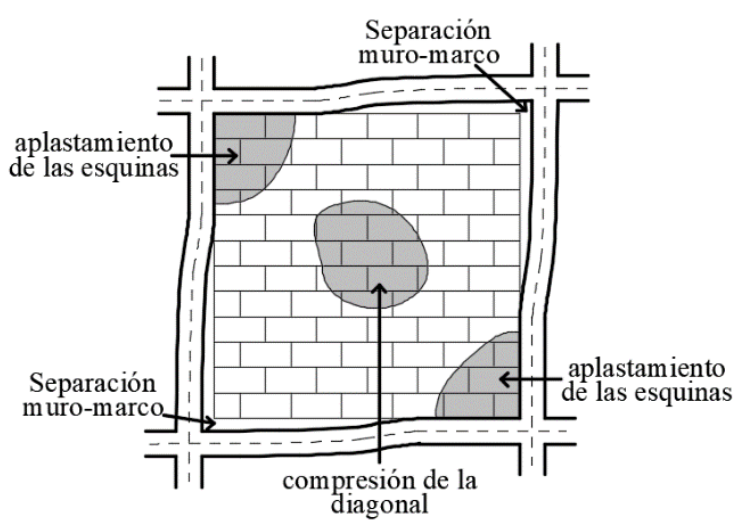

b)

Figura 2. Diferentes modos de falla en marcos rellenos con muros diafragma. a) falla por deslizamiento, tensión diagonal y falla en el muro, b) aplastamiento en las esquinas del muro y falla por compresión de la diagonal

En el Capítulo 4 de las nuevas Normas Técnicas publicadas en diciembre del 2017 (NTCM, 2017) se establecen nuevas recomendaciones para el análisis y diseño de muros diafragma de mampostería, incluyendo un modelo especifico de análisis y nuevas expresiones para el cálculo de su resistencia a corte. Estas nuevas expresiones se adaptaron de las del código canadiense (CSA S304.1-14, 2014), con los parámetros de resistencia de la mampostería usados en la norma mexicana. Para sustentar el uso de estas expresiones se verificó que los resultados de resistencia calculados con dichas fórmulas dan resultados satisfactorios, comparándolos con los resultados de un programa experimental.

En el estudio experimental se ensayaron seis especímenes utilizando como variables de estudio la rigidez relativa muro/marco, el uso de elementos de confinamiento en el muro y el uso de refuerzo horizontal en las juntas de mampostería. Los criterios de selección de las variables fueron: 1) su potencial relevancia en el comportamiento estructural del sistema, 2) la falta de información sobre sus efectos en el comportamiento de los muros diafragma, y 3) su aplicabilidad en la vida profesional.

No se encontró en la literatura revisada información sobre la contribución de los elementos de confinamiento en el comportamiento de muros diafragma. En la mayoría de las ocasiones los muros diafragma son de mampostería simple. Una de las razones para no usar dalas y castillos es que se considera que el marco ya provee confinamiento. Sin embargo, el marco se separa del muro para pequeños desplazamientos laterales, es decir que el efecto de confinamiento se pierde desde etapas tempranas de la respuesta del sistema ante carga lateral. Por otro lado, en muros confinados típicos, donde la rigidez lateral de los castillos es pequeña, comparada con la del muro, los elementos de concreto no suelen separarse del muro, manteniendo su efecto de confinamiento hasta la falla del sistema (Pérez-Gavilán et al. 2015). Otras razones para no utilizar elementos de confinamiento son el costo adicional y la dificultad para construir la dala de cerramiento, la cual debe estar en contacto con la viga del marco. 
Los efectos benéficos que los elementos de confinamiento proporcionan al comportamiento de un muro de mampostería son ampliamente reconocidos. Los principales efectos benéficos de las dalas y los castillos es que incrementan sustancialmente la capacidad de deformación del muro a pesar de que éste sufra un daño importante ante la acción sísmica, y proporcionan un considerable incremento en la estabilidad fuera del plano del muro (Meli, 1973). Los muros de mampostería simple tienen una muy limitada capacidad de distorsión y su resistencia se degrada rápidamente después del primer agrietamiento por tensión diagonal. Otros efectos benéficos de los elementos de confinamiento son, entre otros, que garantizan el contacto entre el muro y el marco ya que estos elementos son colados después de que el muro se construye. Adicionalmente, los castillos permiten la inclusión de refuerzo horizontal, el cual debe ser anclado apropiadamente sin ser traslapado. Debido a que el marco se construye antes que los muros, es difícil proveer refuerzo horizontal en las juntas de mortero sin la inclusión de castillos.

En muros confinados, el refuerzo horizontal incrementa significativamente su resistencia a corte y su capacidad de desplazamiento lateral. Las NCTM (2017) establecen la ecuación 7 para estimar la contribución del refuerzo horizontal en la resistencia a cortante de muros confinados.

$$
V_{S R}=F_{R} \eta p_{h} f_{y h} A_{T}
$$

donde $\eta$ un el factor que toma en cuenta la resistencia a compresión de la mampostería, el área neta de las piezas y el efecto de la cuantía de refuerzo en la resistencia a corte de la mampostería (Pérez-Gavilán y Cruz, 2017; Rubio, 2018). Dicho factor, aunque conserva el símbolo utilizado en las NTCM (2004), ya no representa un factor de eficiencia como se conceptualizaba en dicho código (Aguilar y Alcocer, 2001; Pineda y Alcocer, 2004; Zepeda y Alcocer, 2001; Pérez-Gavilán y Cruz, 2017; Rubio, 2018). Debido a que no se cuenta con datos para el uso de este factor en muros diafragma, las normas mexicanas proponen utilizar los mismos criterios y factores que para muros confinados. La participación del refuerzo horizontal supone una falla por tensión diagonal ya que requiere que el refuerzo cruce las grietas para deformarse. En el modo de falla por deslizamiento, el refuerzo es paralelo a la grieta por lo que no sufre deformaciones importantes. Por esta razón, no se incluye la contribución del refuerzo en ese tipo de falla.

Una importante característica de los muros incluidos en esta investigación es su relación de aspecto $(H / L)$, ya que, en gran parte de la literatura revisada, los muros tuvieron un valor de $H / L$ cercano a la unidad (muros cuadrados) a pesar de que en la práctica es más común observar muros diafragma largos, puesto que los claros de los marcos de concreto o acero están entre los seis y doce metros, y la altura de entrepiso no suele exceder de $3.5 \mathrm{~m}$.

En esta publicación se presenta el desarrollo de las nuevas especificaciones para el análisis, diseño y construcción de muros diafragma incluidas en la nueva edición de las Normas Técnicas Complementarias para el diseño y construcción de estructuras de Mampostería, (NTCM, 2017). Se presenta un ejemplo del funcionamiento de las expresiones, tomando como variables independientes la relación de aspecto y la resistencia a compresión de la mampostería. Los resultados obtenidos utilizando las nuevas expresiones de diseño, se comparan con los resultados de un programa experimental.

\section{CAMBIOS EN LAS EXPRESIONES DE LA RESISTENCIA DE MUROS DIAFRAGMA EN LA NORMA MEXICANA DE MAMPOSTERÍA}

\section{Modelo de análisis}

En las NTCM (2017) se adoptó el modelo de la diagonal equivalente del código canadiense (Figura 3), el cual se basa en el modelo propuesto por Stafford-Smith (1962). En dicho modelo, la sección 
transversal de la diagonal es de un espesor igual al espesor del muro y su ancho se calcula por medio de la ecuación 8.

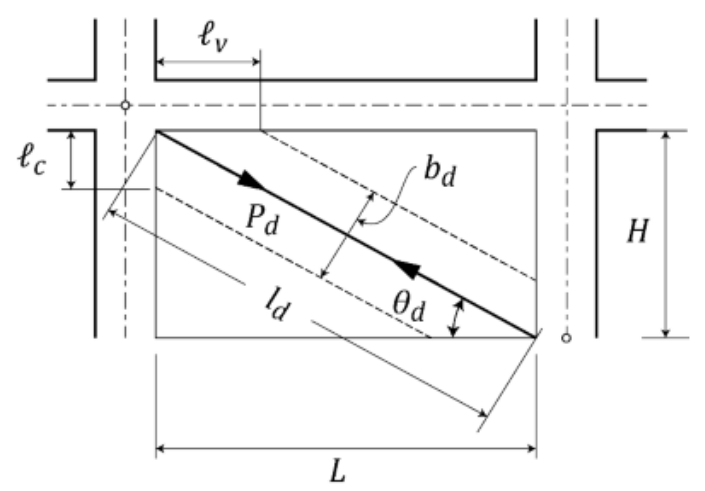

Figura 3. Modelo de diagonal equivalente de las NTCM (2017)

$$
b_{d}=\frac{1}{2} \sqrt{\ell_{c}^{2}+\ell_{v}^{2}} \leq \frac{l_{d}}{4}
$$

Los parámetros $\ell_{c}$ y $\ell_{v}$ son las longitudes de contacto entre el muro y las columnas, y entre el muro y la viga, respectivamente; $l_{d}$ es la longitud de la diagonal. $\ell_{c}, \ell_{v}$ y $l_{d}$ se determinan con las ecuaciones 9 , 10 y 11 , respectivamente.

$$
\begin{aligned}
& \ell_{c}=\frac{\pi}{2}\left(\frac{4 E_{f} I_{c} H}{E_{m} t \operatorname{sen} 2 \theta_{d}}\right)^{1 / 4} \\
& \ell_{v}=\pi\left(\frac{4 E_{f} I_{v} L}{E_{m} t \operatorname{sen} 2 \theta_{d}}\right)^{1 / 4} \\
& l_{d}=\left(H^{2}+L^{2}\right)^{1 / 2}
\end{aligned}
$$

donde $E_{f}$ y $E_{m}$ son el módulo de elasticidad del material del marco y de la mampostería, respectivamente; $I_{c}$ y $I_{v}$ son el momento de inercia de la sección transversal bruta de las columnas y la viga; $t, H$ y $L$ son el espesor, la altura libre y la longitud del muro, respectivamente; y $\theta_{d}$ es el ángulo que forma la diagonal del muro con la horizontal.

\section{Nuevas expresiones para la resistencia a corte de muros diafragma}

\section{Resistencia a corte por aplastamiento}

De acuerdo con el código canadiense, la resistencia a compresión de la diagonal equivalente se calcula mediante la ecuación 12

$$
P_{r}=\left(0.85 \chi \phi_{m} f_{m}^{\prime}\right) \cdot A_{e}
$$

donde $\phi_{m}$ es un factor de reducción de resistencia, similar al factor $F_{R}$ utilizado en las normas mexicanas; $f_{m}^{\prime}$ es la resistencia a compresión de diseño de la mampostería en dirección normal a las juntas de mortero; $\chi$ es un factor que se utiliza para tomar en cuenta la dirección de los esfuerzos de compresión en un miembro 
de mampostería relativo a la dirección usada para determinar $f_{m}^{\prime}$ (para muros diafragma se considera igual a 0.50$)$; y $A_{e}$ es el área transversal efectiva de la diagonal equivalente igual a $b_{d} \cdot t$.

En las NTCM (2017) se define la resistencia a corte por aplastamiento $\left(V_{R a}\right)$ como la componente horizontal de la resistencia a compresión de la diagonal, es decir $V_{R a}=P_{r} \cos \left(\theta_{d}\right)$. Sustituyendo cada parámetro en la ecuación 12 , se tiene que

$$
\frac{V_{R a}}{\cos \left(\theta_{d}\right)}=\left(0.85 \cdot 0.50 \cdot F_{R} \cdot f_{m}^{\prime}\right) \cdot b_{d} \cdot t \quad \rightarrow \quad V_{R a}=\left(0.425 \cdot F_{R} \cdot f_{m}^{\prime}\right) \cdot b_{d} \cdot t \cdot \cos \left(\theta_{d}\right)
$$

siendo 2.5\% más conservadoras, las NTCM (2017) incluyen la ecuación 13 para estimar la resistencia a corte por aplastamiento.

$$
V_{R a}=0.4 F_{R} f_{m}^{\prime} b_{d} t \cdot \cos \left(\theta_{d}\right)
$$

\section{Fuerza cortante resistente por deslizamiento}

La resistencia al deslizamiento, de acuerdo con el CSA S304.1-14, se estima con la ecuación 14

$$
V_{R S}=0.16 \phi_{m} \sqrt{f_{m}^{\prime}} A_{u c}+\phi_{m} \mu P_{1}
$$

En la ecuación 14, el valor $0.16 \sqrt{f_{m}^{\prime}}$ representa la resistencia a tensión diagonal de la mampostería, que en México está representada por la cantidad $0.50 v_{m}^{\prime} ; A_{u c}$ es el área no agrietada de la sección transversal del muro, la cual se considera igual al 80 por ciento del área transversal del muro $\left(A_{u c}=0.8 A_{T}\right) ; \mu$ es el coeficiente de fricción igual a 1.0 para juntas de mortero; y $P_{1}$ es la fuerza de compresión actuando normal al plano de deslizamiento. Para muros diafragma, $P_{1}$ se toma como $90 \%$ de la componente vertical $\left(P_{v}\right)$ de la fuerza de compresión resultante de la acción del puntal $\left(P_{1}=0.9 P_{v}\right)$. En la condición de falla por deslizamiento, $P_{v}=V_{R S} \tan \left(\theta_{d}\right)$.

En las NTCM (2017) se denomina a la resistencia al deslizamiento como la resistencia a corte por deslizamiento $\left(V_{R d}\right)$, es decir $V_{R d}=V_{R S}$. Sustituyendo cada parámetro en la ecuación 14, se tiene que

$V_{R d}=0.5 v_{m}^{\prime} \cdot F_{R} \cdot 0.8 A_{T}+F_{R} \cdot 1.0 \cdot 0.9 V_{R d} \tan \left(\theta_{d}\right)$

La expresión anterior conduce a la ecuación 15 para estimar la resistencia cortante por deslizamiento de un muro diafragma de mampostería, incluida en las NTCM (2017)

$$
V_{R d}=\frac{0.4 v_{m}^{\prime} F_{R} A_{T}}{1-0.9 F_{R} \tan \left(\theta_{d}\right)}
$$

En la primera edición de las NTCM (2017) existe una errata en la ecuación 4.4.1 donde aparece un coeficiente de 0.5 en vez de 0.4 .

\section{$\underline{\text { Resistencia a tensión diagonal }}$}

Para calcular la resistencia a tensión diagonal en muros diafragma, las NTCM (2017) consideran las mismas ecuaciones que para muros confinados. Éstas establecen que la resistencia a tensión diagonal de un muro de mampostería $\left(V_{R t}\right)$ es igual la fuerza cortante resistida por la mampostería $\left(V_{m R}\right)$ más la fuerza 
cortante resistida por el refuerzo horizontal $\left(V_{S R}\right)$. El cálculo de $V_{m R}$ y $V_{S R}$ se realiza por medio de las ecuaciones 16 y 17 , respectivamente.

$$
\begin{aligned}
& V_{m R}=F_{R}\left[\left(0.5 v_{m}^{\prime} A_{T}+0.3 P\right) \cdot f\right] \leq 1.5 F_{R} v_{m}^{\prime} A_{T} f \\
& V_{S R}=F_{R} \eta p_{h} f_{y h} A_{T}
\end{aligned}
$$

donde $f$ es un factor que toma en cuenta la relación de aspecto $(H / L)$ del muro, igual a 1.5 si $H / L \leq 0.2$, 1.0 si $H / L \geq 1.0$ y para valores de $H / L$ entre 0.2 y 1.0 se interpola linealmente (Pérez-Gavilán et al. 2015); $\mathrm{P}$ es la carga axial que actúa sobre el muro la cual se considera igual a cero para muros diafragma; $p_{h}$ es la cuantía de refuerzo horizontal; $f_{y h}$ es el esfuerzo de fluencia del acero de refuerzo; y $\eta$ es un factor que toma en cuenta la resistencia a compresión de la mampostería, el área neta de las piezas y el efecto de la cuantía de refuerzo en la resistencia a corte de la mampostería, como se dijo anteriormente, y se calcula con las ecuaciones 18, 19, 20 y 21.

$$
\begin{aligned}
\eta & =\frac{V_{m R}}{F_{R} p_{h} f_{y h} A_{T}}\left(k_{0} k_{1}-1\right)+\eta_{s} \\
k_{0} & =\left\{\begin{array}{ll}
1.3 \text { si } H / L \leq 1.0 \\
1.0 & \text { si } H / L \geq 1.5
\end{array} \quad \text { (para valores intermedios de } H / L,\right. \text { se interpola linealmente) } \\
k_{1} & =1-\alpha p_{h} f_{y h} \\
\eta_{s} & =\left\{\begin{array}{ll}
0.75 & \text { si } f_{m}^{\prime} \geq 9 \mathrm{MPa} \\
0.55 & \text { si } f_{m}^{\prime} \leq 6 \mathrm{MPa}
\end{array} \text { (para valores intermedios de } f_{m}^{\prime},\right. \text { se interpola linealmente) }
\end{aligned}
$$

En la ecuación $20, k_{1}$ no debe ser menor que $1-0.1 f_{a n} f_{m}^{\prime} \alpha$, donde $f_{a n}$ es el cociente entre el área neta y el área bruta de las piezas y $\alpha=0.45 \mathrm{MPa}^{-1}$. Si $p_{h} f_{y h}>0.1 f_{a n} f_{m}^{\prime}$, el valor de $\eta_{s}$ de la ecuación 21 deberá multiplicarse por $0.1 f_{a n} f_{m}^{\prime} / p_{h} f_{y h}$.

\section{Discusión de las nuevas expresiones para la resistencia a corte de muros diafragma}

A diferencia del código canadiense, en las normas mexicanas el formato seleccionado de las expresiones permite relacionar los diferentes modos de falla con distintos valores de resistencia a corte de muros diafragma. Esta forma de expresar los resultados de las expresiones permite una comparación directa de los tres modos de falla.

Se presenta a continuación un ejemplo del funcionamiento de las expresiones de resistencia a corte para distintos valores de relación de aspecto y distintos valores de resistencia a compresión de la mampostería. Los valores supuestos de las propiedades de los materiales y algunos parámetros geométricos del marco se presentan en la Tabla 1.

Para lograr expresar las ecuaciones 15 y 16 en términos de $f_{m}^{\prime}$ es necesario relacionar dicho valor con la resistencia a compresión diagonal de diseño de la mampostería $\left(v_{m}^{\prime}\right)$. En la ¡Error! No se encuentra el origen de la referencia. se presentan los valores medios de la resistencia a compresión $\left(f_{m}\right)$ y la resistencia a compresión diagonal $\left(v_{m}\right)$ para distintos tipos de mampostería, obtenidos en algunas investigaciones experimentales desarrolladas en México. Con base en estos resultados se encontró la ecuación 22 (ver ¡Error! No se encuentra el origen de la referencia.). 
Tabla 1. Propiedades mecánicas de los materiales y geometría del marco

\begin{tabular}{|c|c|c|}
\hline Parámetro & Unidades & Definición \\
\hline$f_{m}^{\prime}$ & $\mathrm{MPa}$ & $\begin{array}{l}\text { Resistencia a compresión de diseño de la mampostería. Se } \\
\text { toma como variable independiente }\end{array}$ \\
\hline$v_{m}^{\prime}=0.18 \sqrt{f_{m}^{\prime}}$ & MPa & $\begin{array}{c}\text { Resistencia a compresión diagonal de diseño de la } \\
\text { mampostería (ver justificación de esta expresión más } \\
\text { adelante). }\end{array}$ \\
\hline$f_{c}^{\prime}=25$ & $\mathrm{MPa}$ & Resistencia a compresión de diseño del concreto en el marco \\
\hline$E_{f}=4400 \sqrt{f_{c}^{\prime}}$ & MPa & Módulo de elasticidad del concreto \\
\hline$E_{m}=600 f_{m}^{\prime}$ & $\mathrm{MPa}$ & Módulo de elasticidad de la mampostería \\
\hline$H=3.00$ & $\mathrm{~m}$ & Altura del muro \\
\hline$t_{w}=0.12$ & $\mathrm{~m}$ & Espesor del muro \\
\hline$w=H / L$ & & $\begin{array}{c}\text { Relación de aspecto del muro. Se toma como variable } \\
\text { independiente. Ya que } H \text { tiene un valor fijo, } L \text { se obtiene a } \\
\text { partir de esta expresión para un } w \text { dado }\end{array}$ \\
\hline$z=h_{c} / h_{v}=0.85$ & & $\begin{array}{l}\text { Cociente entre la dimensión de la columna paralelo al plano } \\
\text { del marco }\left(h_{c}\right) \text { y el peralte de la viga }\left(h_{v}\right)\end{array}$ \\
\hline$b_{c}=h_{c}$ & $\mathrm{~m}$ & $\begin{array}{l}\text { Dimensión de la columna perpendicular al plano del marco. } \\
\text { Se asume que la columna es cuadrada }\end{array}$ \\
\hline$h_{v}=\left(L_{m}+h_{c}\right) / 10$ & $\mathrm{~m}$ & Peralte de la viga, igual a un décimo del claro del marco \\
\hline$b_{v}= \begin{cases}0.25 & \text { si } h_{v}<0.4 \\
0.40 & \text { si } h_{v}>2.0\end{cases}$ & $\mathrm{m}$ & $\begin{array}{c}\text { Ancho de la viga. Para valores de } h_{v} \text { entre } 0.4 \text { y } 2 \text {, se } \\
\text { interpola linealmente }\end{array}$ \\
\hline
\end{tabular}

$$
v_{m}=0.32 \sqrt{f_{m}}
$$

la cual se expresa en MPa. La ecuación 22 relaciona aproximadamente $f_{m}$ y $v_{m}$. Para tener una idea de esta relación para valores de diseño, se sustituyeron las definiciones de los valores de diseño de estos parámetros de acuerdo a las NTCM (2017) (ecuaciones 23 y 24), en la ecuación 22.

$$
\begin{aligned}
& v_{m}^{\prime}=\frac{v_{m}}{1+2.5 c_{v}} \\
& f_{m}^{\prime}=\frac{f_{m}}{1+2.5 c_{f}}
\end{aligned}
$$

Utilizando los valores mínimos de los coeficientes de variación $c_{v}=0.2$ y $c_{f}=0.15$, conduce a la ecuación 25

$$
v_{m}^{\prime}=0.18 \sqrt{f_{m}^{\prime}}
$$




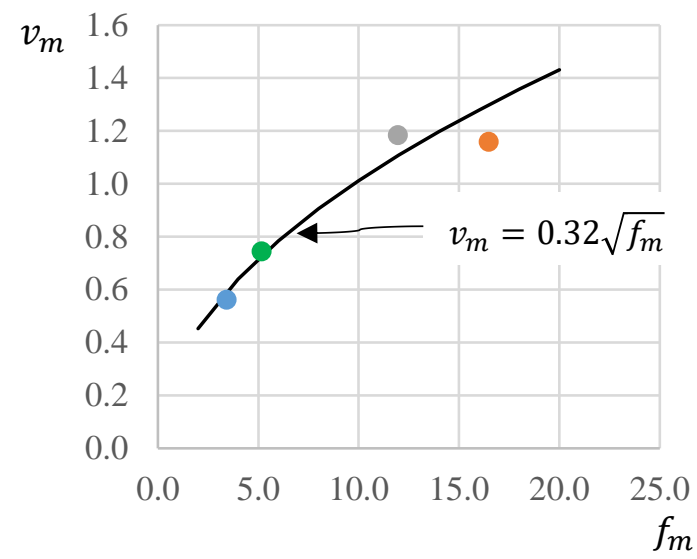

- Tabique rojo recocido

- Block de concreto, $12 \times 20 \times 40 \mathrm{~cm}$ (Leal, 2010)

- Block de concreto multiperforado (Pérez-Gavilán y Cruz, 2017)

- Piezas extruidas (Rubio, 2018)

Figura 4. Gráficas resistencia a compresión diagonal (MPa) - resistencia a compresión (MPa) de diferentes tipos de mampostería (valores medios)

En la Figura 5 se presenta se presenta la variación del ancho de la diagonal equivalente en función de la relación de aspecto del muro $(H / L)$, utilizando la ecuación 8.

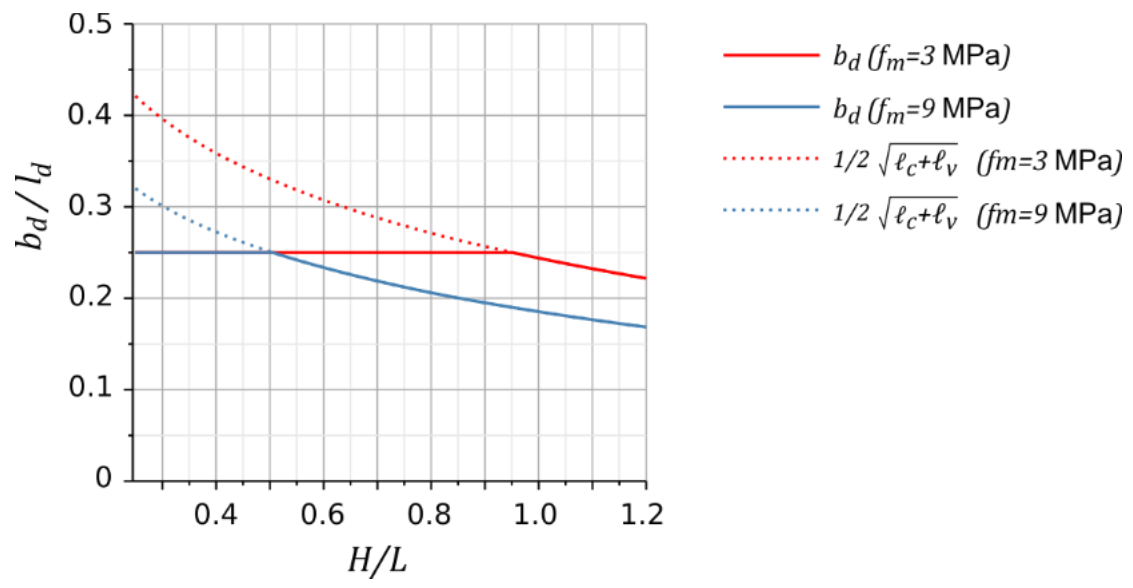

Figura 5. Ancho de la diagonal equivalente normalizada con respecto a la longitud de la diagonal relación de aspecto $(H / L)$

En la Figura 6a se muestra el esfuerzo cortante resistente, $\tau$, para los distintos modos de falla de los muros diafragma, representados en el ejemplo, en función de la relación de aspecto. Se incluyen dos grupos de curvas: las curvas con líneas discontinuas se calcularon con un valor de $f_{m}^{\prime}=9 \mathrm{MPa}$ y las curvas con líneas continuas con $f_{m}^{\prime}=3 \mathrm{MPa}$; El valor de $\tau$ se calculó como el cociente entre la resistencia a corte del muro y su área transversal, $A_{T}$. En la Figura 6a se observa que para el caso de una mampostería de escasa resistencia a compresión $\left(f_{m}^{\prime}=3 \mathrm{MPa}\right)$, la falla es controlada, en muros largos hasta $H / L=0.8$, por el deslizamiento. Para valores de $H / L$ mayores a 0.8 , domina la falla por aplastamiento. Para el caso de una mampostería de mayor resistencia a compresión $\left(f_{m}^{\prime}=9 \mathrm{MPa}\right)$, el deslizamiento domina la falla hasta un valor de la relación de aspecto poco menor de $H / L=1$; para valores mayores de $H / L$, domina el aplastamiento. En ningún caso domina la falla por tensión diagonal. En todos los casos de la Figura 6a, el muro incluye refuerzo horizontal. 
En la Figura 6b se presenta la variación del esfuerzo cortante resistente, $\tau$, para los distintos modos de falla, en función de la resistencia a compresión de diseño de la mampostería, para un valor fijo igual a 0.7 de la relación de aspecto. Las curvas con líneas continuas corresponden a un muro sin refuerzo horizontal. La resistencia a corte por tensión diagonal resulta menor a la resistencia por deslizamiento. El resultado depende del valor supuesto de $v_{m}^{\prime}$. La curva con línea discontinua corresponde a un muro con refuerzo horizontal. En ese caso, la resistencia tensión diagonal aumenta significativamente por lo que ya no domina la falla. Para muros con mampostería de baja resistencia a compresión, $f_{m}^{\prime}<2 \mathrm{MPa}$, el aplastamiento domina la falla.

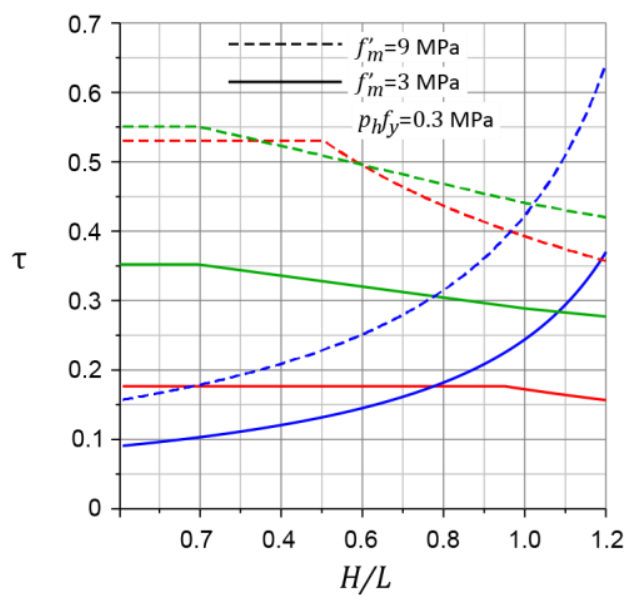

a)

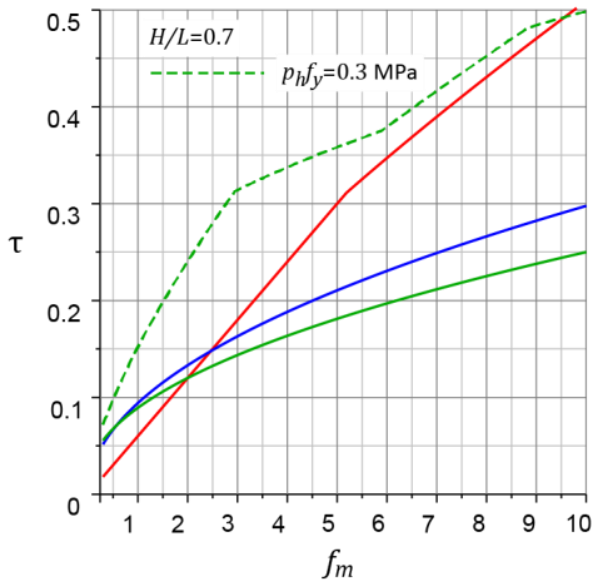

b)

Figura 6. Esfuerzo cortante resistente para los distintos modos de falla de los muros diafragma representados en el ejemplo de la Tabla 1

\section{PROGRAMA EXPERIMENTAL}

\section{Diseño de los especímenes}

\section{Configuración de los especímenes}

Los especímenes se diseñaron con base en el análisis y diseño de dos edificios prototipo de tres y seis niveles, con una altura de entrepiso de $3 \mathrm{~m}$, y tres ejes con $7 \mathrm{~m}$ de claro cada dirección. Se construyeron seis especímenes a escala 1:2 en pares (Figura 7). Los especímenes en cada par tuvieron idénticas características excepto por el tamaño de los elementos del marco, ya que en un caso representaron a los elementos del marco del edificio prototipo de tres niveles mientras que en el otro correspondieron a los elementos del marco del edificio prototipo de seis niveles.

En el primer par de especímenes no se incluyeron elementos de confinamiento ni refuerzo horizontal (especímenes MD3NSR y MD6NSR). En el segundo par, se agregaron dalas y castillos al muro diafragma (especímenes MD3N, y MD6N). Debido a la relación de aspecto del muro, se incluyó un castillo central. Finalmente, en el tercer par, se agregó refuerzo horizontal en las juntas de mortero y se ancló a los castillos (especímenes MD3NRH y MD6NRH). 

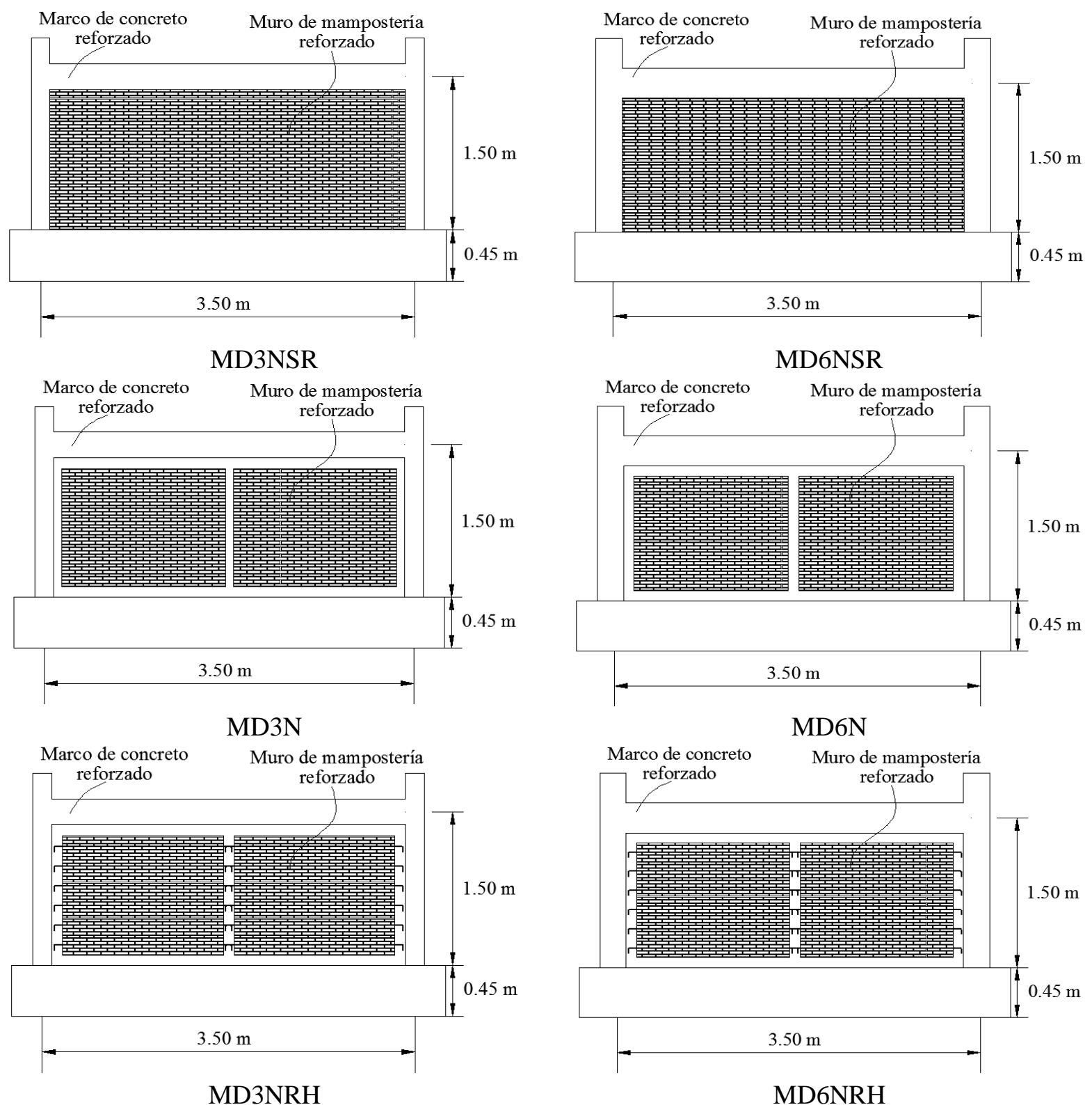

Figura 7. Especímenes de prueba

\section{$\underline{\text { Refuerzo }}$}

En la Figura 8 y la Figura 9 se presentan detalles del refuerzo y dimensiones de los marcos de los especímenes correspondientes a los edificios prototipo de tres y seis niveles, respectivamente. El refuerzo longitudinal en columnas y vigas consistió en barras de acero Grado 42 de 12.7 mm (No. 4) y 9.5 mm (No. 3) de diámetro con estribos de alambrón de $6.4 \mathrm{~mm}$ (No. 2) de diámetro. En las columnas, la cuantía de refuerzo fue $3.3 \%$ en especímenes de columnas de tamaño menor mientras que en especímenes de columnas de tamaño mayor fue $2.4 \%$. El espaciamiento de los estribos fue reducido en los extremos de los elementos ya que se podrían desarrollar articulaciones plásticas en esas zonas. El refuerzo horizontal en el muro consistió en una barra de acero Grado 60 de $4.0 \mathrm{~mm}$ de diámetro colocada a cada seis hiladas (Figura 10). 


\section{Geometría de los muros}

Las dimensiones globales del muro correspondientes al edificio prototipo de tres niveles fueron 1375 $\mathrm{mm}$ de altura y $3325 \mathrm{~mm}$ de longitud, teniendo una relación de aspecto igual a 0.414. Las dimensiones globales del muro correspondientes al edificio prototipo de seis niveles fueron $1350 \mathrm{~mm}$ de altura y 3250 $\mathrm{mm}$ de longitud, teniendo una relación de aspecto $H / L$ igual a 0.415 . Las dimensiones nominales de las piezas, de tabiques rojo recocido, fueron $25 \times 65 \times 125 \mathrm{~mm}$ y las juntas de mortero tuvieron un espesor igual a $7 \mathrm{~mm}$. Los detalles del refuerzo y dimensiones en los muros se muestran en la Figura 10.

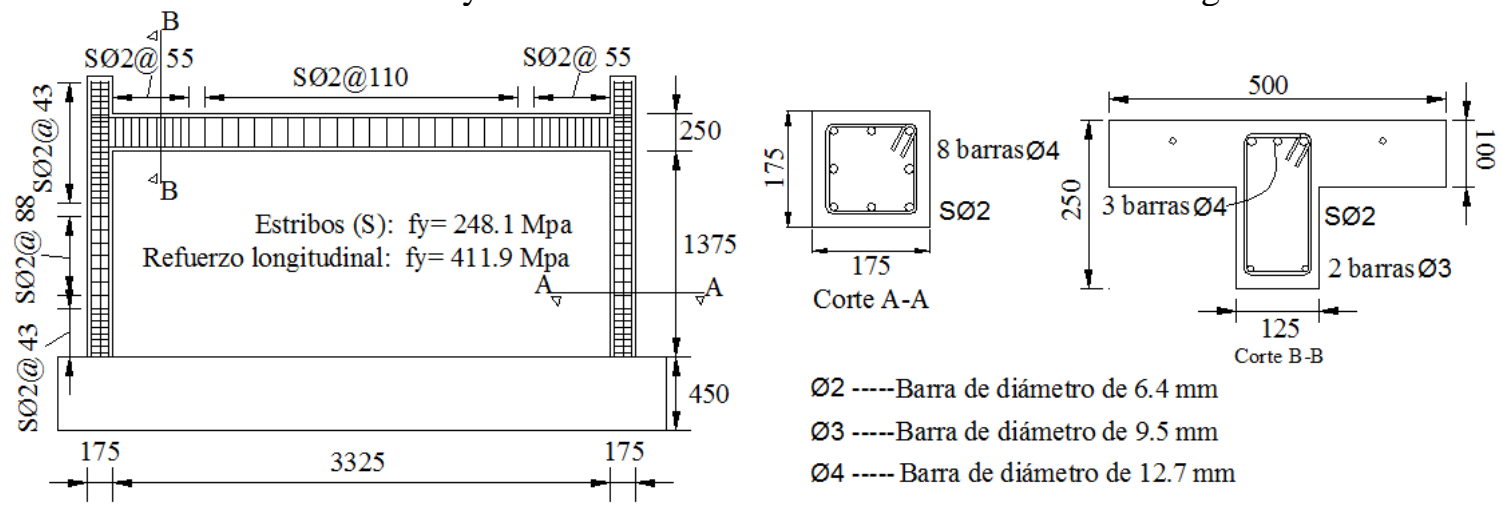

Figura 8. Detalles del refuerzo y dimensiones (en milímetros) del marco de los especímenes MD3NSR, MD3N y MD3NRH
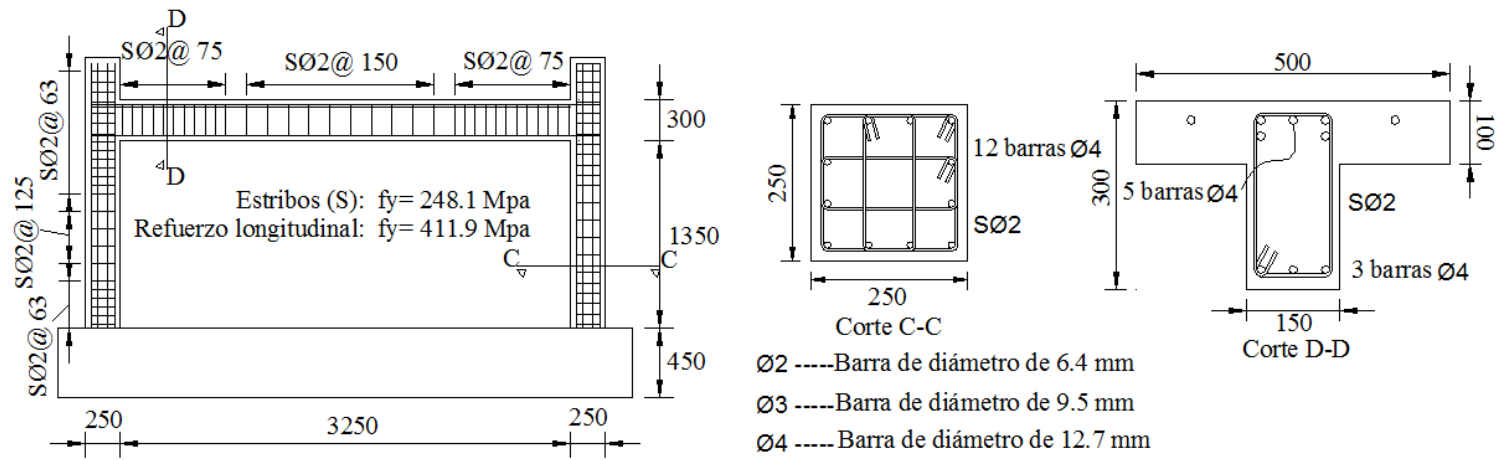

Figura 9. Detalles del refuerzo y dimensiones (en milímetros) del marco de los especímenes MD6NSR, MD6N y MD6NRH

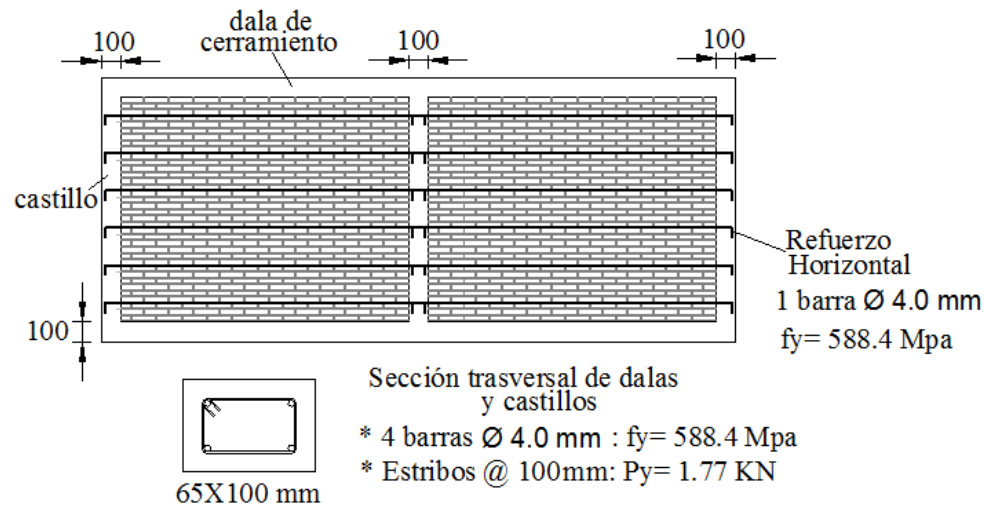

Figura 10. Detalles del refuerzo y dimensiones de los muros MD3NRH y MD6NRH 


\section{Propiedades de los materiales}

Cada una de las propiedades mecánicas de los materiales se obtuvo a partir de la norma del Organismo Nacional de Normalización y Certificación de la Construcción y Edificación (ONNCCE) correspondiente. Los resultados obtenidos en general tuvieron una baja dispersión. Debido a que los especímenes se construyeron en pares, las propiedades de los materiales corresponden a ambos especímenes en el par. En la Tabla 2 se presenta un resumen de los valores promedio de las propiedades de los materiales correspondientes a cada par de especímenes. En la Tabla 3 se presentan las propiedades mecánicas del acero, el cual se obtuvo de un mismo lote para la construcción de todos los especímenes.

Tabla 2. Propiedades promedio de los materiales. Unidades: MPa

\begin{tabular}{|c|c|c|c|c|c|c|c|c|c|}
\hline \multirow{2}{*}{ Espécimen } & \multicolumn{2}{|c|}{$\begin{array}{l}\text { Concreto en el } \\
\text { marco }\end{array}$} & \multicolumn{2}{|c|}{$\begin{array}{c}\text { Concreto en } \\
\text { dalas y castillos }\end{array}$} & \multicolumn{3}{|c|}{ Mampostería } & \multirow{2}{*}{$\frac{\text { Piezas }}{f_{p}}$} & \multirow{2}{*}{$\frac{\text { Mortero }}{f_{j}}$} \\
\hline & $f_{c f}^{\prime}$ & $E_{c f}$ & $f_{c c}^{\prime}$ & $E_{c c}$ & $f_{m}$ & $v_{m}$ & $E_{m}$ & & \\
\hline $\begin{array}{l}\text { MD3NSR } \\
\text { MD6NSR }\end{array}$ & 26.12 & 22402 & - & - & 3.49 & 0.63 & 725 & 9.36 & 16.82 \\
\hline $\begin{array}{l}\text { MD3N } \\
\text { MD6N }\end{array}$ & 19.03 & 19490 & 15.07 & 17220 & 3.4 & 0.53 & 703 & 9.36 & 15.01 \\
\hline $\begin{array}{l}\text { MD3NRH } \\
\text { MD6NRH }\end{array}$ & 19.95 & 19719 & 13.3 & 15661 & 3.34 & 0.59 & 693 & 9.36 & 8.01 \\
\hline
\end{tabular}

Tabla 3. Propiedades mecánicas del acero. Unidades: MPa

\begin{tabular}{cccc}
\hline Varillas de acero & $F_{y}$ & $E_{s}$ & $F_{\text {máx }}$ \\
\hline $\begin{array}{c}\text { Refuerzo en el marco. varilla de } \\
12.7 \mathrm{~mm}\end{array}$ & 438 & 194196 & 676 \\
$\begin{array}{c}\text { Refuerzo en las juntas de } \\
\text { mortero. varilla de } 4.0 \mathrm{~mm}\end{array}$ & 644 & 188688 & 719 \\
\hline
\end{tabular}

\section{Construcción de los especímenes}

Los especímenes se construyeron sobre vigas de cimentación de concreto reforzado de $0.45 \times 1.00 \times 4.20 \mathrm{~m}$, las cuales tuvieron aberturas verticales cerca de los extremos para anclar las columnas del marco (Figura 11a). Después de que el espécimen se ensayó, el concreto en las aberturas y el acero de refuerzo de las columnas se removieron para reutilizar la viga en la construcción de los siguientes especímenes.

En todos los casos, el marco se construyó primero, y después de siete días, el marco se rellenó con un muro de mampostería. Monolíticamente con la viga del marco, se construyó una losa de $100 \mathrm{~mm}$ de espesor para conectar al espécimen con la viga de acero utilizada en el sistema para aplicar la carga lateral. Para los muros con elementos de confinamiento, se construyó una dala de desplante sobre la cimentación, en la cual se anclaron los castillos del muro (Figura 11b). Los castillos se colaron después de que la mampostería se construyó. Para garantizar que el marco estuviera en contacto con el muro, se construyó una dala de cerramiento con el sistema de colado que se muestra en la Figura 11c. Se vertió concreto con un revenimiento mayor a $180 \mathrm{~mm}$ a través de ventanas en la cimbra, mismas que se observan en la Figura 11c. El concreto acumulado en dichas ventanas proporcionó la presión hidrostática suficiente para rellenar completamente la dala de cerramiento. Una vez que el concreto fraguó, el exceso se removió mediante un dispositivo mecánico de corte. En la parte superior de cada columna, se ancló una placa de $19.1 \mathrm{~mm}$ de 
espesor para conectar el sistema usado para aplicar la carga vertical. En los especímenes sin elementos de confinamiento, el muro se construyó directamente sobre la cimentación y la mampostería se puso en contacto con el marco con el mismo mortero que se utilizó en las juntas.

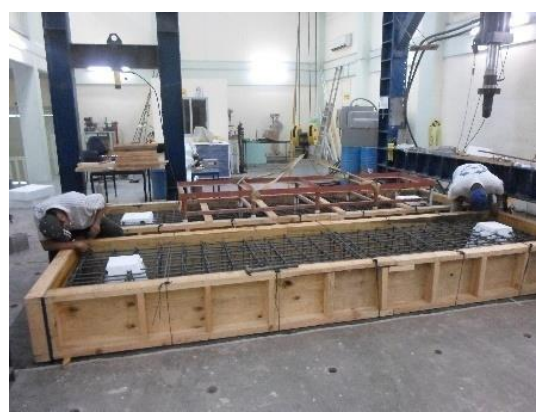

a)

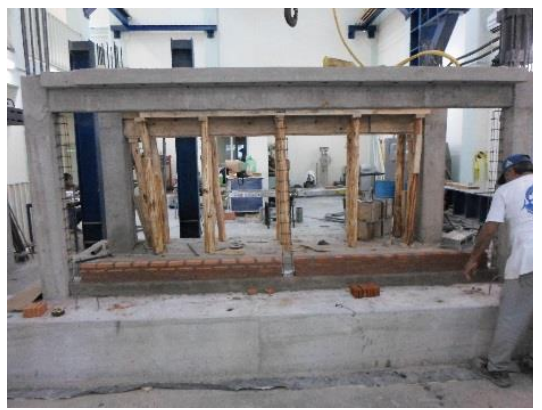

b)

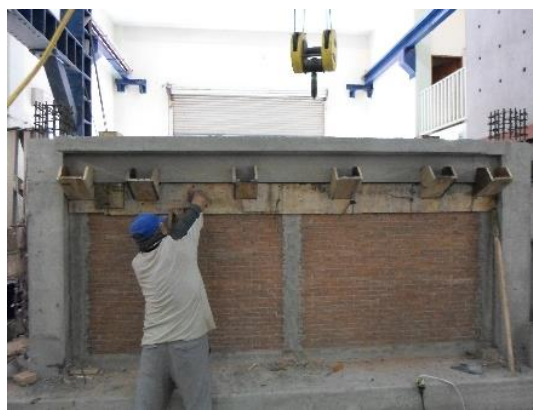

c)

Figura 11. Construcción de los especímenes, a) construcción de la cimentación, b) construcción del espécimen, c) colado de la dala de cerramiento

\section{Marco de carga}

El marco de carga que se utilizó durante los ensayes se muestra en la Figura 12. La carga vertical se aplicó directamente sobre las columnas con actuadores hidráulicos, los cuales reaccionaron contra una viga de acero anclada a la cimentación mediante tensores a cada lado del muro (Figura 12b). La magnitud de las acciones fue la correspondiente a las cargas gravitacionales de los pisos superiores y la carga muerta del piso inferior. El peso de la viga de acero colocada sobre la viga del marco, usada para transferir la carga lateral al espécimen, correspondió a la carga viva del piso inferior. Para simular la transmisión de la carga sísmica por medio del sistema de piso, en forma distribuida, la carga lateral se aplicó a una viga de acero, la cual fue atornillada a la viga del marco (Figura 12a).

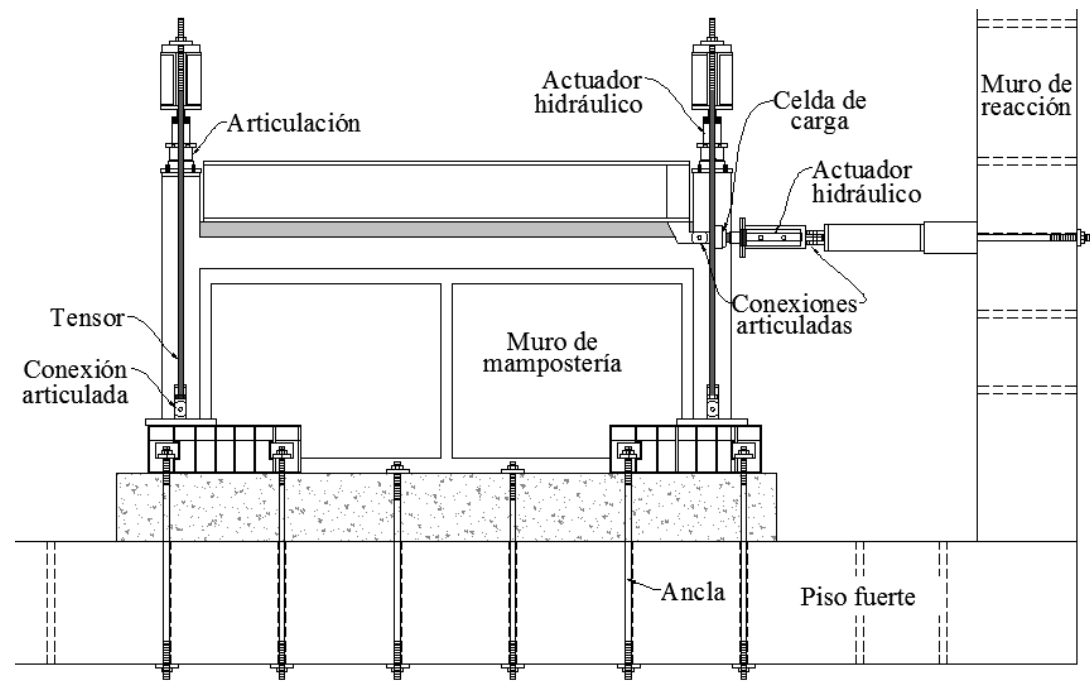

a)

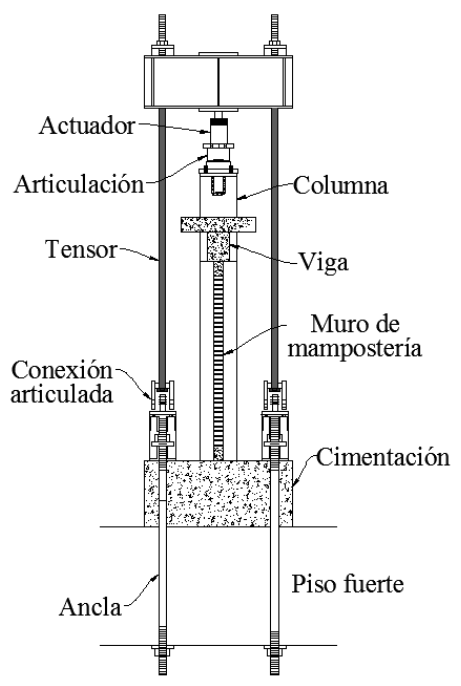

b)

Figura 12. Marco de carga, a) vista en elevación, b) vista en corte 


\section{Secuencia de ensaye}

Los ensayes iniciaron con la aplicación de la carga vertical sobre las columnas, la cual se mantuvo constante durante el ensaye. La magnitud de la carga se determinó a partir de un análisis por carga gravitatoria de los correspondientes edificios prototipo. El hecho de aplicar la carga vertical directamente sobre las columnas se justifica de acuerdo a las consideraciones siguientes: 1) el marco y el sistema de piso se construyen antes que los muros, por lo que el peso de los pisos superiores se transfiere a las columnas y 2) las columnas son axialmente más rígidas que los muros. En estudios previos (Crisafulli, 1997 y Mehrabi et al., 1996) se tomaron en cuenta consideraciones similares, utilizando marcos de carga similares. Se realizó un análisis elástico utilizando un modelo de elemento finito de los dos edificios prototipo; los resultados demostraron que las columnas en el piso inferior soportan más del $98 \%$ de la carga viva total.

La magnitud de la carga vertical en cada columna de los especímenes correspondientes a los edificios prototipo de tres y seis pisos fue $120.17 \mathrm{kN}$ y $245.20 \mathrm{kN}$, respectivamente. El peso de la viga de acero sobre el espécimen fue $12.75 \mathrm{kN}$. Con el objetivo de preservar los esfuerzos por carga vertical, de acuerdo a las leyes de similitud, la carga en los modelos fue igual a una cuarta parte de la calculada para el edificio prototipo. Antes de iniciar el ensaye, se estimó la resistencia al agrietamiento del muro igual a $60 \mathrm{kN}$. La carga lateral se aplicó siguiendo una secuencia similar a la establecida en el protocolo de ensaye descrito en el Apéndice A de las NTCM (2017), el cual se describe en la Figura 13.

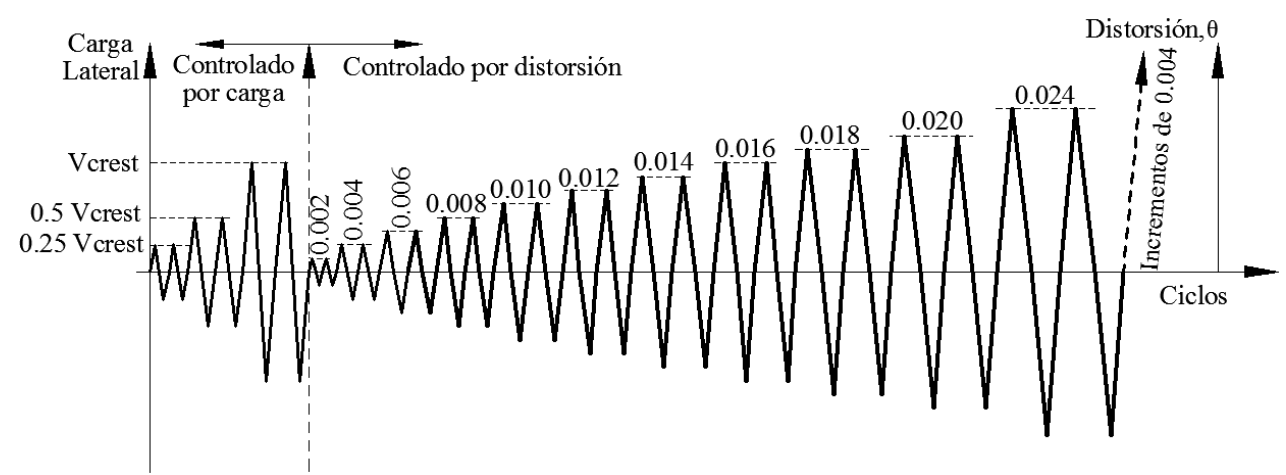

Figura 13. Protocolo de ensaye

Los primeros dos ciclos fueron controlados por carga hasta el $25 \%$ de la carga de agrietamiento estimada; los siguientes dos ciclos hasta el 50\% de la carga de agrietamiento estimada y dos más hasta el $100 \%$ de la carga de agrietamiento. Subsecuentemente, los ensayes fueron controlados por desplazamiento. Se aplicaron distorsiones, desplazamiento lateral entre la altura $(\gamma=\delta / H)$, con incrementos de 0.002 , haciendo dos ciclos por cada incremento.

Cuando el espécimen alcanzó una distorsión de 0.02, el incremento de distorsión cambió a 0.004. Este cambio no se encuentra especificado en las NTCM (2017), ya que este procedimiento fue diseñado principalmente para muros de mampostería confinada, no para muros diafragma.

\section{Instrumentación}

Los especímenes se instrumentaron debidamente para poder registrar las cargas aplicadas, los desplazamientos laterales y las deformaciones en el acero de refuerzo horizontal en los especímenes que lo incluían. Además, se midieron las deformaciones en el refuerzo longitudinal en tres secciones transversales de las columnas y una sección de la viga. En la Figura 14 y la Figura 15 se presentan la instrumentación interna y externa, respectivamente, para medir los parámetros de interés. 
Con los resultados de los ensayes, se evaluó el comportamiento de los especímenes con base en la resistencia al agrietamiento, la resistencia máxima y las distorsiones laterales correspondientes, así como el registro fotográfico del nivel y tipo de daño observado. La contribución del refuerzo a la resistencia de los muros que lo incluían se determinó estimando la fuerza total desarrollada por el refuerzo en los muros, considerando el comportamiento histerético del refuerzo.

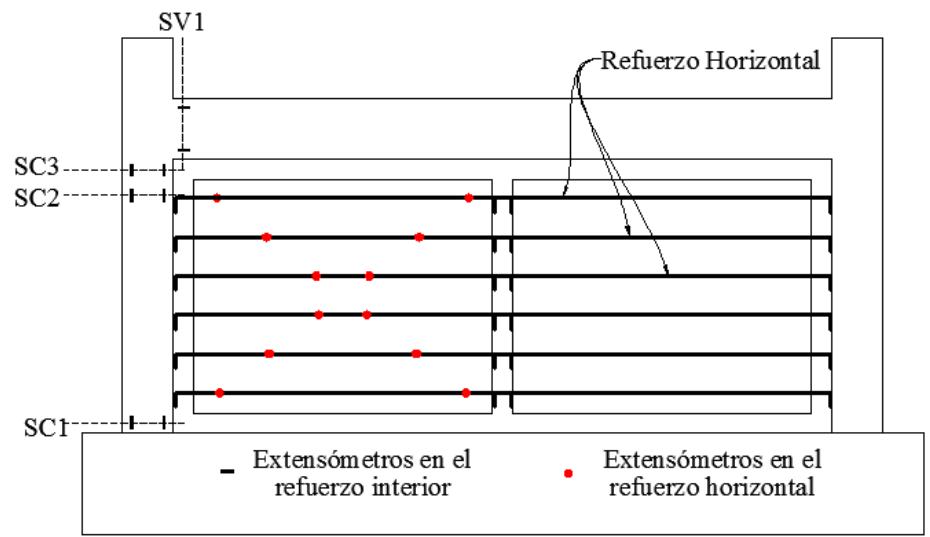

a)

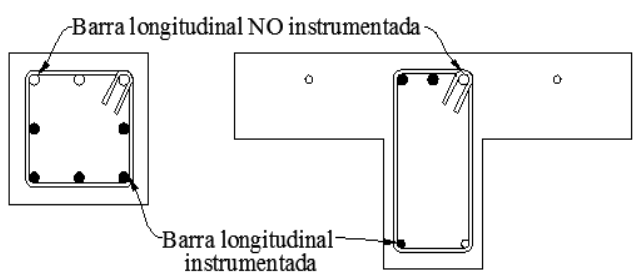

b)

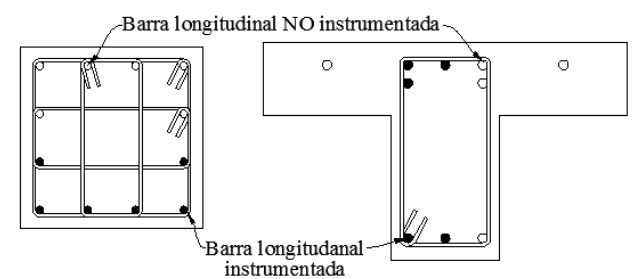

c)

Figura 14. Instrumentación interna, a) ubicación de los extensómetros en los especímenes, b) ubicación de los extensómetros en las secciones de columnas de tamaño menor, c) ubicación de los extensómetros en las secciones de columnas de tamaño mayor

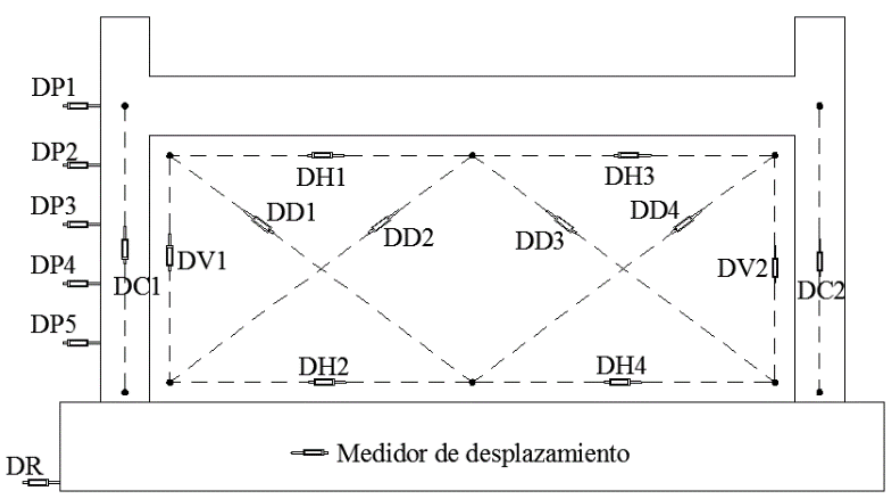

Figura 15. Instrumentación externa 


\section{RESULTADOS EXPERIMENTALES}

\section{Resistencia y deformación}

En la Figura 16a se presenta la curva histerética del especien MD3N, la cual es típica. En todos los casos la estabilidad de los ciclos fue muy buena, a juzgar por lo bien que el ciclo de repetición se aproximó al primer ciclo en cada incremento de desplazamiento. Los especímenes tuvieron una degradación de resistencia muy lenta, como se observó en los ensayes realizado por otros autores (Crisafulli, 1997 y Mehrabi et al., 1996). También se presentó un efecto de estrechamiento ("pinching") de la rama de descarga y se observa que la rama de carga intersecta el eje vertical, aproximadamente, al mismo nivel de carga lateral.

En la Figura 16b se presenta la curva envolvente correspondiente, incluyendo sus puntos críticos: primer agrietamiento $\left(\gamma_{c r}, V_{c r}\right)$, fluencia $\left(\gamma_{y s}, V_{y s}\right)$, resistencia máxima $\left(\gamma_{\max }, V_{\max }\right)$, y falla o resistencia última $\left(\gamma_{U}, V_{U}\right)$. En la Tabla 4 se resume los valores de la distorsión y la carga lateral correspondientes a los puntos críticos.

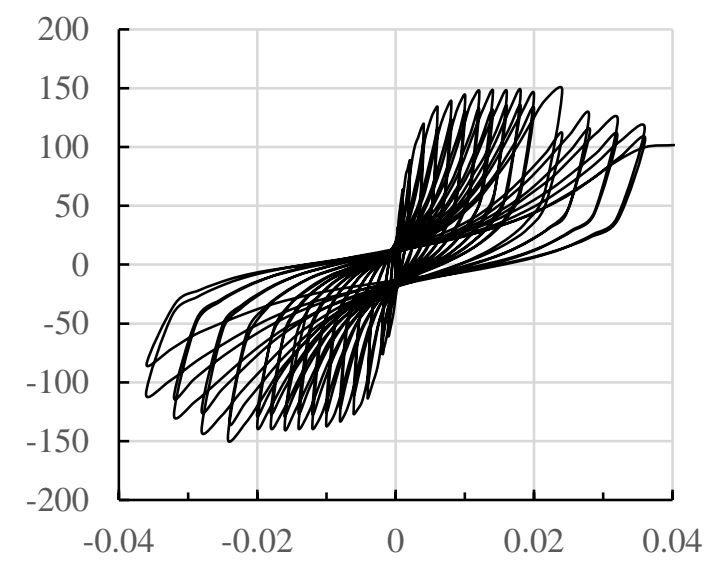

a)

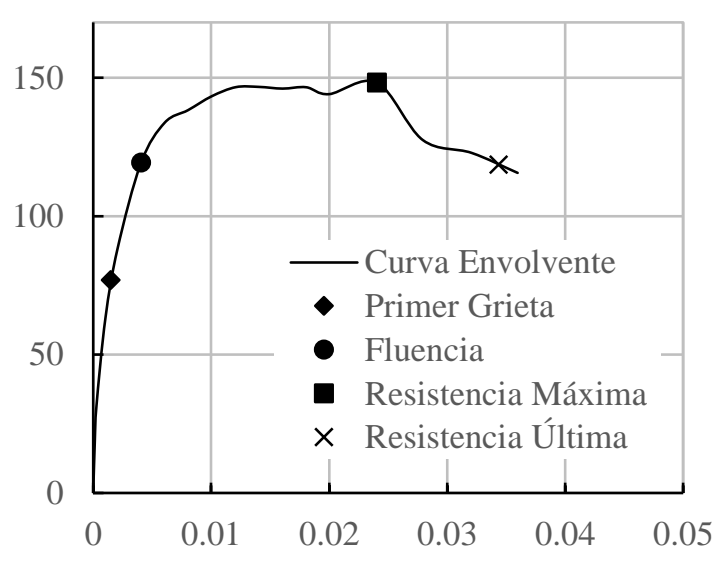

b)

Figura 16. Curvas histeréticas carga lateral $(\mathrm{kN})$ - distorsión $(\mathrm{mm} / \mathrm{mm})$

Usualmente, el primer agrietamiento es asociado a un cambio en la pendiente de la curva envolvente; sin embargo, en estos ensayes, el primer agrietamiento no tuvo un efecto aparente en la gráfica cargadistorsión. La distorsión al agrietamiento $\left(\gamma_{c r}\right)$ fue consistentemente cercana a 0.0014 , la cual coincide con las distorsiones al primer agrietamiento para muros de mampostería confinada reportada en estudios previos (Meli, 1973 y Pérez-Gavilán et al., 2015). Este valor de $\gamma_{c r}$ fue consistente para la mayoría de los especímenes, excepto para el modelo MD3NSR debido a que se desarrolló una separación prematura del muro y el marco. Este fenómeno se explica más adelante, en la discusión de los resultados experimentales. Por esta razón, las distorsiones del espécimen MD3NSR no pueden ser comparadas con las distorsiones de los otros modelos. Consecuentemente, estos valores son excluidos de la comparación y los valores promedio descritos a continuación. La resistencia al agrietamiento promedio de los especímenes con columnas de tamaño menor fue $74.5 \mathrm{kN}$ con un rango de [68.6, 78.0]; y para los especímenes con columnas de tamaño mayor, $109.5 \mathrm{kN}$ con un rango de [98.9, 115.4].

El punto de fluencia corresponde al punto en el que se observó un cambio significativo en la pendiente de la curva envolvente. La distorsión a la fluencia fue muy consistente, con un valor igual a 0.0056 . La resistencia a la fluencia promedio de los especímenes con columnas de tamaño menor fue $126.4 \mathrm{kN}$ con un 
rango de valores de $[108.9,150.9]$, mientras que para los especímenes con columnas de tamaño mayor, la resistencia a la fluencia promedio fue de $246.2 \mathrm{kN}$ con un rango de [233.4, 264.7]. La resistencia a la fluencia fue, en promedio, $98.5 \%$ mayor que la resistencia al agrietamiento (rango para $V_{y s} / V_{c r}$ de $[1.40,2.36]$ ).

La distorsión a la resistencia máxima fue también muy consistente. El valor de la distorsión a la resistencia máxima tuvo un valor promedio de 0.026 . La resistencia máxima promedio de los especímenes con columnas de menor tamaño fue de $168.3 \mathrm{kN}$ con un rango de valores de [148.2, 194.0]; y para los especímenes con columnas de mayor tamaño, la resistencia máxima promedio fue $297.6 \mathrm{kN}$ con un rango de [267.7, 313.7]. La resistencia máxima fue, en promedio, $27.5 \%$ mayor que la resistencia a la fluencia con un rango para $V_{\max } / V_{y s}$ de $[1.15,1.49]$, con solo una pequeña diferencia con el tamaño de las columnas. El valor promedio del cociente anterior para los especímenes con columnas de tamaño menor fue 1.34, y para los especímenes con columnas de tamaño mayor fue 1.21 .

La resistencia y deformación a la falla o última de los especímenes $\left(\gamma_{u}, V_{u}\right)$, se definió en aquel punto de la envolvente donde la resistencia del sistema se redujo al $80 \%$ de la resistencia máxima. El promedio global de la distorsión a la falla fue 0.0394 con pequeñas diferencias con el tamaño de las columnas.

Tabla 4. Rigidez inicial y puntos críticos de la curva envolvente

\begin{tabular}{ccccccccc}
\hline \multirow{2}{*}{ Espécimen } & \multicolumn{2}{c}{$\begin{array}{c}\text { Resistencia al } \\
\text { agrietamiento }\end{array}$} & \multicolumn{2}{c}{$\begin{array}{c}\text { Resistencia a la } \\
\text { fluencia }\end{array}$} & \multicolumn{2}{c}{ Resistencia máxima } & \multicolumn{2}{c}{ Resistencia última } \\
\cline { 2 - 9 } & $\gamma_{c r}^{+}$ & $V_{c r}^{+}$ & $\gamma_{y s}^{+}$ & $V_{y s}^{+}$ & $\gamma_{\text {max }}^{+}$ & $V_{\text {max }}^{+}$ & $\gamma_{U}^{+}$ & $V_{U}^{+}$ \\
\hline MD3NSR & $0.0028^{(+)}$ & 78.0 & 0.0040 & 108.9 & 0.0100 & 162.6 & 0.0270 & 130.0 \\
MD3N & 0.0014 & 76.9 & 0.0040 & 119.4 & 0.0241 & 148.2 & 0.0343 & 118.6 \\
MD3NRH & 0.0014 & 68.6 & 0.0060 & 150.9 & 0.0241 & 194.0 & 0.0377 & 155.2 \\
MD6NSR & 0.0014 & 98.9 & 0.0060 & 233.4 & 0.0242 & 267.7 & 0.0442 & 214.2 \\
MD6N & 0.0016 & 115.4 & 0.0060 & 240.4 & 0.0335 & 311.4 & 0.0395 & 249.1 \\
MD6NRH & 0.0014 & 114.4 & 0.0060 & 264.7 & 0.0240 & 313.7 & 0.0412 & 251.0 \\
\hline
\end{tabular}

${ }^{(+)}$debido a contracciones del mortero de la junta entre muro y marco

\section{Agrietamiento}

Se desarrollaron patrones de agrietamiento similares en los seis especímenes ensayados. El agrietamiento en los especímenes se asoció a cuatro etapas de la curva envolvente definidas por los puntos críticos (Figura 17).

En la primera etapa, la separación entre muro y marco se manifestó por medio de grietas en la interface de estos elementos. La separación máxima de $18 \mathrm{~mm}$ se observó en el espécimen MD3NSR. No se observó separación entre la viga de cimentación y el muro, ni entre el muro y la viga del marco; sin embargo, se observó deslizamiento a todo lo largo de la interface muro-viga, iniciando desde las primeras etapas del ensaye. No se observó separación entre los elementos de confinamiento y el muro.

En los especímenes sin elementos de confinamiento, la primera grieta diagonal, a una distorsión de 0.0014, se desarrolló en la zona central del muro, mientras que en el caso de los especímenes con dalas y castillos en la zona central de uno de los paneles. Los especímenes sin elementos de confinamiento desarrollaron una primera grieta inclinada bien definida, mientras que los especímenes con dala y castillos 
desarrollaron grietas en el muro que fueron más distribuidas. Los especímenes con refuerzo horizontal desarrollaron el agrietamiento mayormente distribuido, en comparación con aquellos especímenes que no incluían refuerzo.

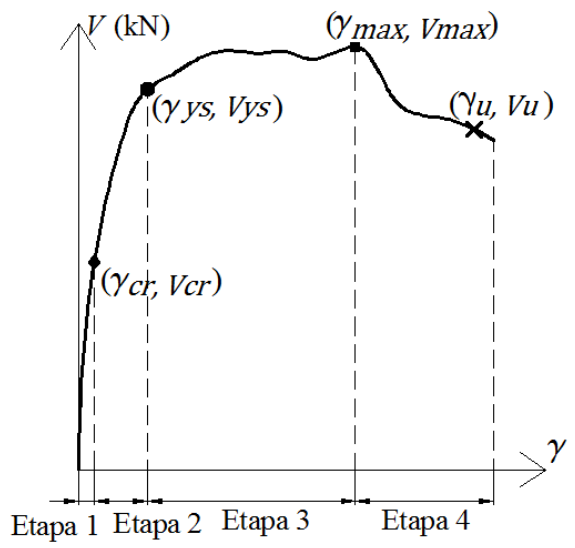

Figura 17. Etapas de agrietamiento

En todos los casos, se desarrollaron grietas en los extremos de la viga del marco, casi simultáneamente con primer agrietamiento inclinado. El promedio de la distorsión al agrietamiento en la viga fue 0.0012.

La segunda etapa de la curva envolvente corresponde a la aparición de grietas de flexión al pie de las columnas. Estas grietas se desarrollaron cuando el muro estaba en contacto con dicha zona del marco, es decir, los momentos de flexión fueron mayores en las equinas de contacto. A medida que el ensaye avanzó, las grietas de flexión al pie de las columnas y el agrietamiento en los extremos de las vigas se extendieron hacia la zona central de los elementos.

La fluencia en las curvas carga lateral - distorsión no ocurrió cuando se detectó el primer agrietamiento, fue hasta que la primera grieta diagonal se extendió y alcanzó los extremos del muro que la fluencia en las curvas envolventes se pudo observar, a una distorsión promedio de 0.0056.

En la tercera etapa, se desarrolló en los muros una combinación de grietas inclinadas y deslizamientos horizontales. Este agrietamiento causó una reducción significativa de la rigidez lateral del sistema. Sin embargo, la carga siguió aumentando hasta alcanzar la resistencia máxima. A medida que el ensaye progresó, los deslizamientos horizontales predominaron, impidiendo que se desarrollaran grietas inclinadas adicionales. En el caso de los especímenes con elementos de confinamiento, los planos de deslizamiento se desarrollaron a cada seis hiladas, los cuales, para muros con refuerzo horizontal, no coincidieron con los planos correspondientes a las juntas con refuerzo horizontal. En los especímenes MD3NSR y MD6NSR, solo dos planos principales de deslizamientos se formaron a la altura media del muro.

$\mathrm{Al}$ aumentar la distorsión, el marco tuvo una participación mayor en la resistencia del sistema, lo cual se reflejó en la aparición de grietas diagonales en la conexión viga-columna. La fluencia en el refuerzo longitudinal de las columnas se alcanzó a una distorsión de 0.012 en los especímenes con columnas de tamaño menor, mientras que en especímenes con columnas de tamaño mayor la fluencia se registró a 0.010 de distorsión. Los deformímetros registraron que la fluencia ocurrió primero al pie de la columna, lo cual concuerda con que el agrietamiento por flexión se observó primero en aquella zona.

En los especímenes con elementos de confinamiento, la resistencia a corte del castillo intermedio tuvo un efecto importante en la resistencia lateral máxima de sistema ( $\gamma_{\max }$ promedio igual a 0.026). 
Durante los ensayes se observó que cuando se aplicó el ciclo correspondiente a la resistencia máxima, el castillo intermedio falló, mediante una grieta que lo cruzó.

La cuarta etapa se caracterizó por el proceso de degradación de la resistencia, el cual incluyó el deterioro de los planos de deslizamiento, la propagación de las grietas en los extremos de la viga y en la base de las columnas y el aplastamiento de la mampostería, especialmente en las esquinas del muro. Esta degradación del sistema ocurrió gradualmente a medida que la distorsión se iba incrementando.

En todos los especímenes, el modo dominante de falla del sistema fue una combinación de deslizamiento y tensión diagonal en el muro, y falla en la conexión viga-columna del marco. El patrón final de agrietamiento del espécimen MD3NRH se muestra en la Figura 18. Se incluye un detalle de la falla en la conexión viga-columna.

Se observó que, después de la falla en el plano, la estabilidad lateral del muro estaba comprometida, especialmente en aquellos especímenes sin elementos de confinamiento; sin embargo, debido a que no se aplicaron cargas fuera de plano, no hubo evidencias de este fenómeno en las gráficas carga - distorsión.

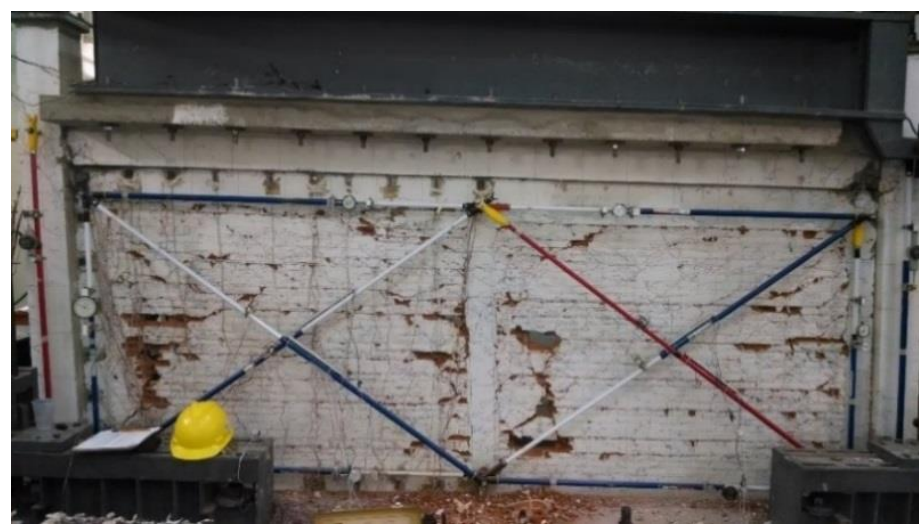

a)

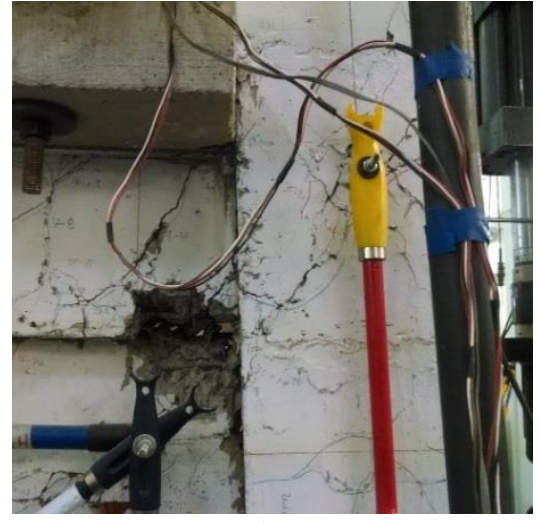

b)

Figura 18. Patrón de agrietamiento final del espécimen MD3NRH, a) agrietamiento en el muro, b) falla en la conexión viga-columna

\section{DISCUSIÓN DE LOS RESULTADOS EXPERIMENTALES}

\section{Efectos de la rigidez relativa muro/marco en el comportamiento del sistema}

En la Figura 19 se presentan las envolventes de las curvas carga lateral - distorsión de los especímenes con rigidez relativa muro/marco distinta. Las gráficas muestran que el marco contribuye significativamente a la resistencia lateral del sistema. En promedio, los especímenes con columnas de tamaño mayor tuvieron, una resistencia al agrietamiento 1.48 veces mayor, una resistencia a la fluencia 1.97 veces mayor, y una resistencia máxima 1.79 veces más grande, que la de los especímenes con columnas de tamaño menor.

El tamaño de las columnas tuvo un efecto en el agrietamiento del muro. En los especímenes con columnas de tamaño mayor el deslizamiento fue predominante. El deslizamiento reduce la formación de nuevas grietas por tensión diagonal, por lo que el daño se concentra en los planos de deslizamiento. 


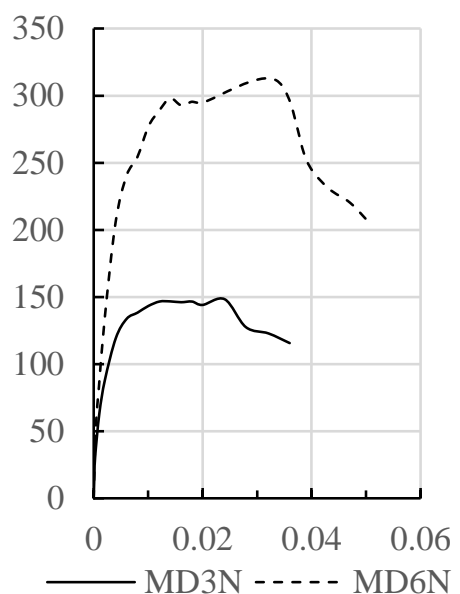

Figura 19. Curvas envolventes, carga lateral $(\mathrm{kN})$ - distorsión $(\mathrm{mm} / \mathrm{mm})$, de especímenes con rigidez relativa muro/marco distinta

\section{Efectos de los elementos de confinamiento en el comportamiento del sistema}

El uso de elementos de confinamiento no afectó significantemente la resistencia a corte del sistema (Figura 20). Este resultado es consistente con lo observado en muros de mampostería confinada en los que los castillos no contribuyen o contribuyen muy poco a su resistencia a corte.

No se observó un efecto significativo en la capacidad de desplazamiento del sistema debida al uso de elementos de confinamiento. Sin embargo, los elementos de confinamiento contribuyeron a la estabilidad fuera del plano de los muros. Esta observación es cualitativa, ya que no hubo una medida numérica para cuantificar este efecto.

El uso de elementos de confinamiento garantiza el contacto entre muro y marco. Crisafulli (1997) reconoció que cuando el marco se construye primero y después se rellena con un muro de mampostería, la contracción en el material del muro o defectos en la construcción pueden causar una falta de contacto inicial. Dicho fenómeno se presentó en esta investigación. El espécimen MD3NSR desarrolló una grieta de contracción en la interface muro/columna en ambos lados del muro. Esta grieta tuvo un considerable efecto en la curva carga-desplazamiento como se observa en la Figura 21, donde se muestran los terceros ciclos de las curvas de los especímenes MD3NSR y MD3N. El lazo del espécimen MD3NSR inicia con una muy baja pendiente, ya que el marco tomó casi toda la carga porque no estaba en contacto directo con el muro. Cuando el marco alcanzó al muro, se observa claramente un abrupto cambio de pendiente a un desplazamiento lateral de alrededor de $0.6 \mathrm{~mm}$.

El hecho de no incluir elementos de confinamiento en el muro, como en el caso de los especímenes MD3NSR y MD6NSR, aumenta la posibilidad del volteo del muro. Una alternativa para asegurar a muros sin confinamiento ante cagas fuera del plano es el uso de elementos a tipo ángulos que conectan al muro y al marco. Esta opción se incluye en las normas mexicanas.

\section{Efectos del refuerzo horizontal en el comportamiento del sistema}

El refuerzo horizontal incrementó considerablemente la resistencia máxima a carga lateral del sistema para el caso de los especímenes con columnas de tamaño menor. Para el caso de los especímenes con columnas de tamaño mayor, no se observó un cambio significativo en la resistencia al corte máxima (Figura 20). 


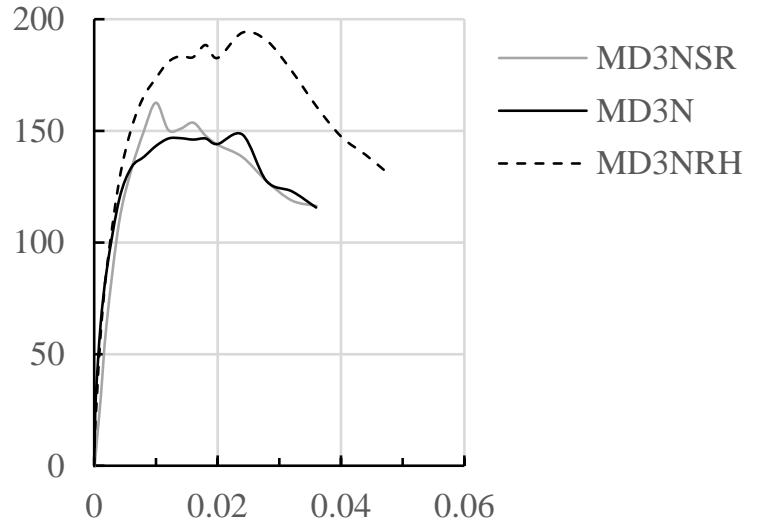

a)

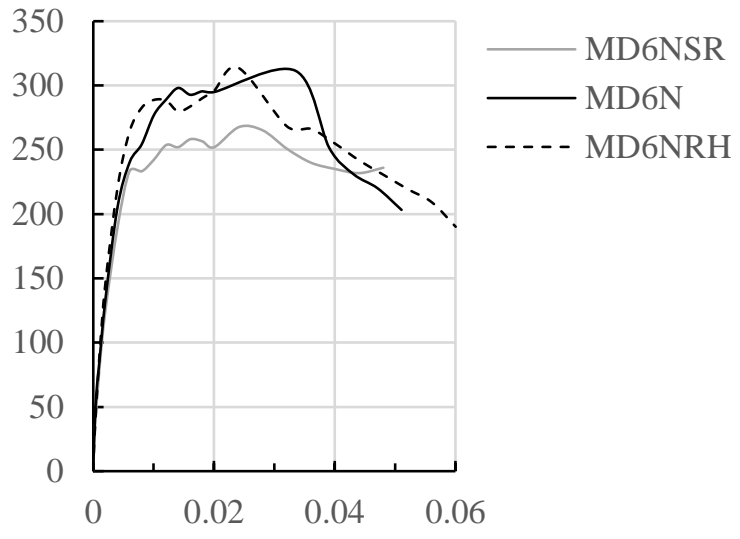

b)

Figura 20. Curvas envolventes, carga lateral $(\mathrm{kN})$ - distorsión $(\mathrm{mm} / \mathrm{mm})$, a) especímenes con columnas de tamaño menor, b) especímenes con columnas de tamaño mayor

Se calculó la fuerza desarrollada por el refuerzo horizontal $\left(F_{h}\right)$ en los dos especímenes que lo incluían (MD3NRH y MD6NRH). La fuerza $F_{h}$ se obtuvo mediante las deformaciones registradas por los extensómetros colocados en las barras (Figura 14). El esfuerzo en cada barra se calculó mediante la relación esfuerzo - deformación obtenida de las pruebas a los materiales. Se consideró el comportamiento histerético de las barras mediante un modelo constitutivo elastoplástico perfecto.

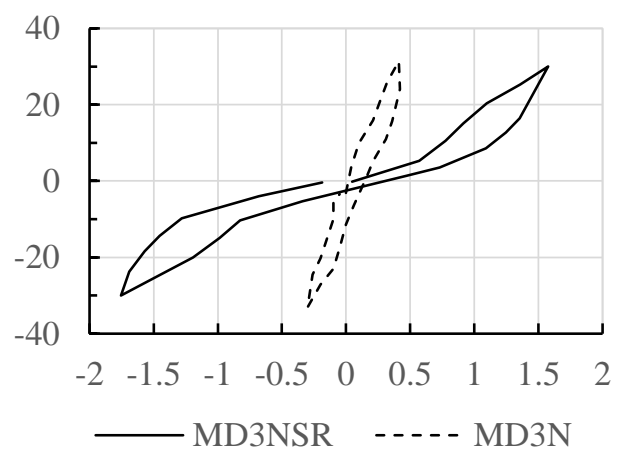

Figura 21. Lazo correspondiente al tercer ciclo de la curva carga lateral $(\mathrm{kN})$ - desplazamiento $(\mathrm{mm})$, de los especímenes MD3NSR y MD3N

En la Figura 22 se presenta la variación de $F_{h}$ con la distorsión. Es evidente que en el espécimen con columnas de tamaño menor la fuerza $F_{h}$ es significativamente mayor que en el caso del espécimen con columnas de tamaño mayor (45.7\% mayor). El valor máximo que alcanzó $F_{h}$ en el espécimen de columnas de tamaño menor y mayor fue $33.25 \mathrm{KN}$ y $22.82 \mathrm{KN}$, respectivamente. La anterior observación es consistente con el hecho de que en el espécimen con columnas de tamaño menor, la contribución del refuerzo a la resistencia global del sistema es mayor, y demuestra, claramente, la mayor eficiencia del refuerzo horizontal. La eficiencia del refuerzo $\left(\eta_{e}\right)$, calculada como la relación del valor máximo de $F_{h}$ y la fuerza en el refuerzo cuando todas las barras alcanzan fluencia, fueron 0.70 y 0.48 , respectivamente.

Las curvas de la Figura 22 presentan un cambio significativo de pendiente en el punto crítico del primer agrietamiento en el muro, lo cual es consistente con el hecho de que el refuerzo horizontal se activa con la primera grieta inclinada. Sin embargo, la contribución del refuerzo al comportamiento global del 
sistema se refleja después del punto de fluencia en el caso del espécimen con columnas de tamaño menor (ver figura 20), mientras que en el espécimen con columnas de tamaño mayor, la contribución del refuerzo se observa en el intervalo de distorsión [0.006, 0.012].

Debido a que el refuerzo horizontal en este tipo de muros requiere de elementos de confinamiento para su anclaje, su inclusión podría representar un aumento significativo en el costo (mayor mano de obra, material y tiempo). Con base en los resultados obtenidos en esta investigación, su aporte a la resistencia depende de la rigidez relativa muro/marco. Se requiere, por lo tanto, un estudio para determinar en qué casos es conveniente incluir o no el refuerzo horizontal, considerando costo-beneficio (incremento de resistencia).

La falta de efecto del refuerzo horizontal en la resistencia máxima de los especímenes con columnas de tamaño mayor se atribuye a que la falla es predominantemente por deslizamiento. Como se explicó anteriormente, el refuerzo horizontal solo puede ser efectivo si grietas inclinadas cruzan las barras horizontales. Esto es reconocido por las NTCM (2017), las cuales no incluyen la contribución del refuerzo horizontal en la resistencia a corte del muro cuando se considera el modo de falla por deslizamiento, mientras que la contribución del refuerzo horizontal sí se considera cuando se asume el modo de falla por tensión diagonal.
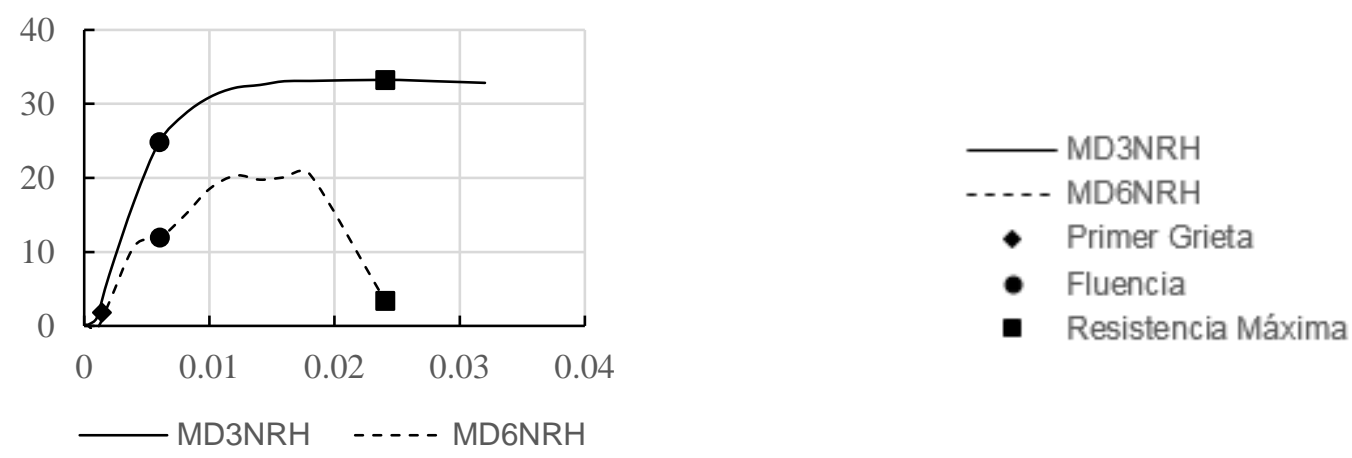

Figura 22. Curvas envolventes fuerza total en el refuerzo $(\mathrm{kN})$ - distorsión $(\mathrm{mm} / \mathrm{mm})$

\section{COMPARACIÓN DE LOS RESULTADOS ANALÍTICOS Y EXPERIMENTALES}

\section{Rigidez lateral}

Se estimó la rigidez lateral de cada espécimen utilizando el modelo de diagonal equivalente establecido en las NTCM (2017). Además, se incluyó la predicción de la rigidez lateral usando los modelos propuestos por Stafford-Smith (1962) y Bazán (1980), y por los códigos MSJC (2010) y NZS 4230 (2004). No se consideró la contribución de los elementos de confinamiento ni el refuerzo horizontal.

Los resultados analíticos se compararon con la rigidez experimental del modelo $\left(K_{b f}\right)$, la cual se calculó como la pendiente de la línea que conecta el origen con el punto de fluencia de la curva envolvente del espécimen correspondiente. Los resultados se presentan en la Tabla 5, incluyendo la variación global promedio con respecto a los resultados experimentales para cada modelo de análisis. Se observa que, en general, con el modelo establecido en el código canadiense y las normas mexicanas se obtuvo la mejor aproximación a los resultados experimentales de los especímenes, a juzgar que en promedio se obtuvo la menor variación $(24.9 \%)$. 
Tabla 5. Resultados analíticos y experimentales de la rigidez lateral del sistema. Unidades: $\mathrm{kN} / \mathrm{mm}$

\begin{tabular}{|c|c|c|c|c|c|c|}
\hline Espécimen & $\begin{array}{l}\text { Smith } \\
(1962, \\
1966, \\
1967)\end{array}$ & $\begin{array}{l}\text { Bazán } \\
\text { (1980) }\end{array}$ & $\begin{array}{l}\text { MSJC } \\
(2010)\end{array}$ & $\begin{array}{c}\text { New } \\
\text { Zealand } \\
\text { Standard } \\
(2004) \\
\end{array}$ & $\begin{array}{c}\text { Canadian } \\
\text { Standard } \\
(2014) / \\
\text { NTCM }(2017) \\
\end{array}$ & $\begin{array}{c}\text { Rigidez } \\
\text { experimental de } \\
\text { sistema }\left(K_{b f}\right)\end{array}$ \\
\hline MD3NSR & 33.7 & 18.2 & 12.1 & 19.4 & 19.0 & 18.1 \\
\hline MD3N & 31.2 & 16.3 & 10.7 & 17.9 & 17.5 & 19.9 \\
\hline MD3NRH & 31.1 & 16.4 & 10.8 & 17.8 & 17.4 & 16.8 \\
\hline MD6NRH & 63.8 & 45.6 & 36.4 & 42.9 & 41.8 & 25.9 \\
\hline MD6N & 57.9 & 40.1 & 32.0 & 38.3 & 37.3 & 26.7 \\
\hline MD6NRH & 58.0 & 40.5 & 32.3 & 38.5 & 37.5 & 29.4 \\
\hline \% variación & 97.9 & 30.7 & 30.9 & 27.2 & 24.9 & \\
\hline
\end{tabular}

Sin embargo, al comparar los resultados por grupos de especímenes, se observa que para los especímenes con columnas de menor tamaño, se obtuvo la mejor aproximación a los resultados experimentales (variación promedio igual a 6.9\%) con las NTCM (2017); mientras que con el MSJC (2010) se obtuvo la mejor aproximación a la $K_{b f}$ de los especímenes con columnas de tamaño mayor, con una variación promedio igual a $23.3 \%$.

\section{Resistencia a carga lateral}

Se estimó la resistencia lateral del sistema con base en las especificaciones de las NTCM (2017). Dichas especificaciones se pueden resumir como sigue: el sistema se modela como un marco arriostrado por un elemento diagonal que representa al muro. Las propiedades de la diagonal se describieron con antelación en este documento; El análisis del sistema es elástico; la resistencia lateral se estima como la carga requerida para que la componente horizontal de la fuerza en la diagonal equivalente alcance la resistencia a cortante en el muro. La resistencia a cortante en el muro se obtuvo como el menor valor entre $V_{R a}, V_{R d}$ y $V_{R t}$.

Además, con el propósito de comparar las nuevas especificaciones de las normas mexicanas con las de la edición anterior, también se estimó la resistencia lateral del sistema con especificaciones de las NCTM (2004). En la edición 2004 de las normas mexicanas no se establece un modelo específico de análisis. En este estudio se utilizó el modelo de diagonal equivalente de las NTCM (2017).

Los resultados analíticos se muestran en la Tabla 6, incluyendo el porcentaje de variación con respecto a los resultados experimentales para las predicciones de cada espécimen. Un valor positivo en el porcentaje de variación indica que se sobreestima la resistencia máxima del sistema.

Se observa que las NTCM (2004) no predicen apropiadamente la resistencia lateral del sistema, en ninguno de los casos. La edición 2004 de las normas mexicanas sobreestiman la resistencia. La variación es significativamente mayor para los especímenes con refuerzo horizontal. Las normas sobreestiman un 22.2\% y $26.5 \%$ la resistencia de los especímenes MD3N y MD6N, respectivamente; mientras que, para el caso de los especímenes con refuerzo, MD3NRH y MD6NRH, la resistencia se sobreestima un $84.3 \%$ y $149.7 \%$, respectivamente. Este hecho se explica debido a que, de acuerdo a las NTCM (2004) la contribución del refuerzo se debe incluir, independientemente del modo de falla en el muro. Esto hace que la resistencia del muro con refuerzo sea elevada, y en consecuencia también la del sistema. 
Tabla 6. Resultados analíticos y experimentales de la resistencia lateral del sistema. Unidades: kN

\begin{tabular}{ccccccc}
\hline \multirow{2}{*}{ Espécimen } & \multicolumn{2}{c}{ NTCM (2004) } & \multicolumn{3}{c}{ NTCM (2017) } & Resultados \\
\cline { 2 - 6 } & $V_{R}$ & Error (\%) & $V_{R}$ & Error (\%) & $\begin{array}{c}\text { Modo de falla } \\
\text { del muro }\end{array}$ & experimentales \\
\hline MD3NSR & 227.1 & 39.7 & 156.8 & -3.5 & Aplastamiento & 162.6 \\
MD3N & 181.2 & 22.2 & 135.7 & -8.5 & Deslizamiento & 148.2 \\
MD3NRH & 357.7 & 84.3 & 143.9 & -25.8 & Aplastamiento & 194.0 \\
MD6NSR & 508.1 & 89.8 & 350.7 & 31.0 & Aplastamiento & 267.7 \\
MD6N & 394.0 & 26.5 & 295.7 & -5.0 & Deslizamiento & 311.4 \\
MD6NRH & 783.3 & 149.7 & 315.0 & 0.4 & Aplastamiento & 313.7 \\
\hline
\end{tabular}

Las NTCM (2017) predicen apropiadamente la resistencia lateral de los especímenes en la mayoría de los casos. Para los especímenes MD3NSR, MD3N, MD6N y MD6NRH el porcentaje de error fue menor a 10\%. Sin embargo, el caso del espécimen MD3NRH la predicción fue 25.8\% menor.

De acuerdo con las normas, el aplastamiento de la mampostería y deslizamiento en las juntas horizontales rigen la falla de los muros (Tabla 6). Con una diferencia menor al $10 \%$ entre los valores de $V_{R a}$ y $V_{R d}$ de cada muro, en todos los casos. Los resultados experimentales muestran que se desarrollaron grietas diagonales en el muro, pero enseguida se formaron planos horizontales de deslizamiento. Conforme el ensaye progresó, el deslizamiento fue ganando mayor importancia. Además, se desarrolló falla por aplastamiento en las esquinas superiores de muro, aunque en los especímenes con confinamiento, las dalas y los castillos redujeron el aplastamiento del muro.

\section{CONCLUSIONES}

El modelo de diagonal equivalente establecido en la edición 2017 de las NTCM es adecuado para predecir la rigidez lateral del sistema. Esto significa que la demanda de resistencia de muros diafragma cuando se sujetan a carga lateral se puede predecir aproximadamente.

La predicción de la resistencia lateral proporcionada por las NTCM (2017) es adecuada en muchos casos; sin embargo, el efecto del tamaño de las columnas en la contribución del refuerzo horizontal requiere ser comprendida mejor.

Un mayor tamaño de columnas produce un modo de falla en el cual predomina el deslizamiento en las juntas. El deslizamiento reduce la producción de nuevas grietas inclinadas, por lo que el daño se concentra en los planos de deslizamiento

Los elementos de confinamiento no tienen un efecto considerable en la resistencia a corte o capacidad de desplazamiento del sistema. Sin embargo, los elementos de confinamiento son útiles para anclar el refuerzo horizontal y para garantizar la estabilidad fuera del plano del muro. Además, las dalas y castillos garantizan el contacto entre el muro y el marco.

El refuerzo horizontal contribuye a la resistencia a corte del sistema. Sin embargo, su eficiencia se reduce a medida que el tamaño de las columnas incrementa. La razón de este fenómeno se relaciona a las conclusiones previas: un mayor tamaño de columnas resulta en un modo de falla en el muro 
predominantemente por deslizamiento en las juntas horizontales de mortero, el cual reduce la formación de nuevas grietas inclinadas. Las grietas inclinadas son necesarias para deformar el refuerzo horizontal y, por lo tanto, contribuir a la resistencia.

\section{REFERENCIAS}

Aguilar, G y S M Alcocer (2001), "Efecto del refuerzo horizontal en el comportamiento de muros de mampostería confinada ante cargas laterales", Informe Técnico, Centro Nacional de Prevención de Desastres, México.

Asteris, P G, S T Antoniou, D T Sophianopoulos y C Z Chrysostomou (2011), "Mathematical macromodeling of infilled frames: state of the art", Journal of Structural Engineering, Vol. 137, No. 12, pp 1508-1517. DOI: 10.1061/(ASCE)ST.1943-541X.0000384

Asteris, P G, D J Kakaletsis, C Z Chrysostomou y E E Smyrou (2011), "Failure modes of in-filled frames", Electronic Journal of Structural Engineering, Vol. 11, No. 1, pp 11-20.

Bazán, E (1980), "Muros de mampostería ante cargas laterales", Tesis de doctorado, Universidad Nacional Autónoma de México, México D.F.

Canadian Standard Association CSA (2014), "Design of masonry structures”, S304-14, Ontario, Canada.

Crisafulli, F J (1997), "Seismic behavior of reinforced concrete structures with masonry infills", Tesis de doctorado, University of Carterbury, New Zealand.

El-Dakhakhni, W W, M Elgaaly y A A Hamid (2003), "Three-strut model for concrete masonry-infilled frames", Journal of Structural Engineering, Vol. 129, No 2, pp 177-185. DOI: 10.1061/(ASCE)07339445(2003)129:2(177)

FEMA (1997), "NEHRP commentary on the guidelines for the seismic rehabilitation of buildings", FEMA274, Washington, DC.

FEMA (1998), "Evaluation of earthquake damaged concrete and masonry wall buildings: Basic procedures manual", FEMA-306, Washington, DC.

Flanagan, R D y R M Bennett (2001), "In plane analysis of masonry infills material”, Practice Periodical on Structural Design and Construction, Vol. 6, pp 176-182. DOI: 10.1061/(ASCE)1084$\underline{0680(2001) 6: 4(176)}$

Holmes (1961), "Steel frames with brickwork and concrete infilling", Proceedings of the Institution of Civil Engineers, Vol. 19, pp. 473-478. DOI: 10.1680/iicep.1961.11305

Leal, J M (2010), "Propiedades físicas y mecánicas de la mampostería de block producido en la ciudad de Culiacán", Tesis de licenciatura, Universidad Autónoma de Sinaloa, Culiacán, Sinaloa.

Madia, F C y G A Parsekian (2011), "Modeling a reinforced concrete building frame with infill walls", $11^{\text {th }}$ North American Masonry Conference, Minneapolis, U.S.A.

Mainstone, R J (1974), "Supplementary note on the stiffness and strengths of infilled frames", Building Research Station, Garston, UK.

Masonry Standards Joint Committee (2010), “MSJC code/commentary working draft”, U.S.A.

Mehrabi, A B, P B Shing, M P Schuller y J L Noland (1996), "Experimental evaluation of masonry-infilled R/C frames", Journal of Structural Engineering, American Society of Civil Engineers, Vol. 122, No. 3, pp. 228-237. DOI: 10.1061/(ASCE)0733-9445(1996)122:3(228) 
Meli, R (1973), "Behaviour of masonry walls under lateral loads", 5th World Conference on Earthquake Engineering, Rome, Italy.

New Zealand Standard (2004), "Design of reinforced concrete masonry structures", NZC 4230: Parte 1 y 2.

NTCM (2004), "Normas técnicas complementarias para diseño y construcción de estructuras de mampostería", Gaceta oficial del Distrito Federal, México, D.F.

NTCM (2017), "Normas técnicas complementarias para diseño y construcción de estructuras de mampostería", Gaceta oficial de la Ciudad de México, México.

Pérez-Gavilán, J J, L E Flores y S M Alcocer (2015), “An experimental study of confined masonry walls with varying aspect ratios", Earthquake Spectra, Vol. 31, pp. 945-68. DOI: 10.1193/090712EQS284M

Pérez-Gavilán, J J y A I Cruz (2017), "Shear strength of confined masonry walls with transverse reinforcement", $16^{\text {th }}$ International Brick and Block Masonry Conference, Padua, Italia

Pineda, J A y S M Alcocer (2004), “Comportamiento ante cargas laterales de muros de mampostería confinada reforzados con malla de alambre soldado", Informe Técnico, Centro Nacional de Prevención de Desastres, México.

Polyakov, S V (1956), Masonry in framed buildings: an investigation into the strength and stiffness of masonry infilling, (Godsudarstvenoe Isdatel'stvo Literatury Po Stroidal stvui Architecture. Moscow, 1956), Traducido por G. L. Cairns, 1963. National Lending Library for Science and Technology, Boston.

Rubio, L (2018), “Contribución del refuerzo horizontal a la resistencia a corte de muros confinados de piezas de arcilla extruida", Tesis de maestría, Universidad Nacional Autónoma de México. Ciudad de México, México.

Stafford-Smith, B (1962), "Lateral stiffness of infilled frames", ASCE Journal of Structural Division, Vol. 88, No. 6, pp. 183-199.

Stafford-Smith, B (1966), "Behavior of square infilled frames", Journal of the Structural Division, Vol. 92, No 1, pp. 381-403.

Stafford-Smith, B (1967), "Methods for predicting the lateral stiffness and strength of multi-storey infilled frames", Building Science, Vol. 2, pp. 247-257. DOI: 10.1016/0007-3628(67)90027-8

Stafford-Smith, B y C Carter (1969), "A method of analysis for infilled frames", Proceedings of the Institution of Civil Engineers, Vol. 44, pp. 31-48. DOI: 10.1680/iicep.1969.7290

Zepeda, J A y S M Alcocer (2001), "Comportamiento ante cargas laterales de muros de ladrillo de arcilla perforado y multiperforado", Informe Técnico, Centro Nacional de Prevención de Desastres, México. 